This item was submitted to Loughborough's Research Repository by the author.

Items in Figshare are protected by copyright, with all rights reserved, unless otherwise indicated.

\title{
Testing for seasonal unit roots by frequency domain regression
}

PLEASE CITE THE PUBLISHED VERSION

LICENCE

CC BY-NC-ND 4.0

\section{REPOSITORY RECORD}

Chambers, Markus J., and Joanne S. McGarry. 2019. "Testing for Seasonal Unit Roots by Frequency Domain Regression”. figshare. https://hdl.handle.net/2134/360. 
This item was submitted to Loughborough's Institutional Repository by the author and is made available under the following Creative Commons Licence conditions.

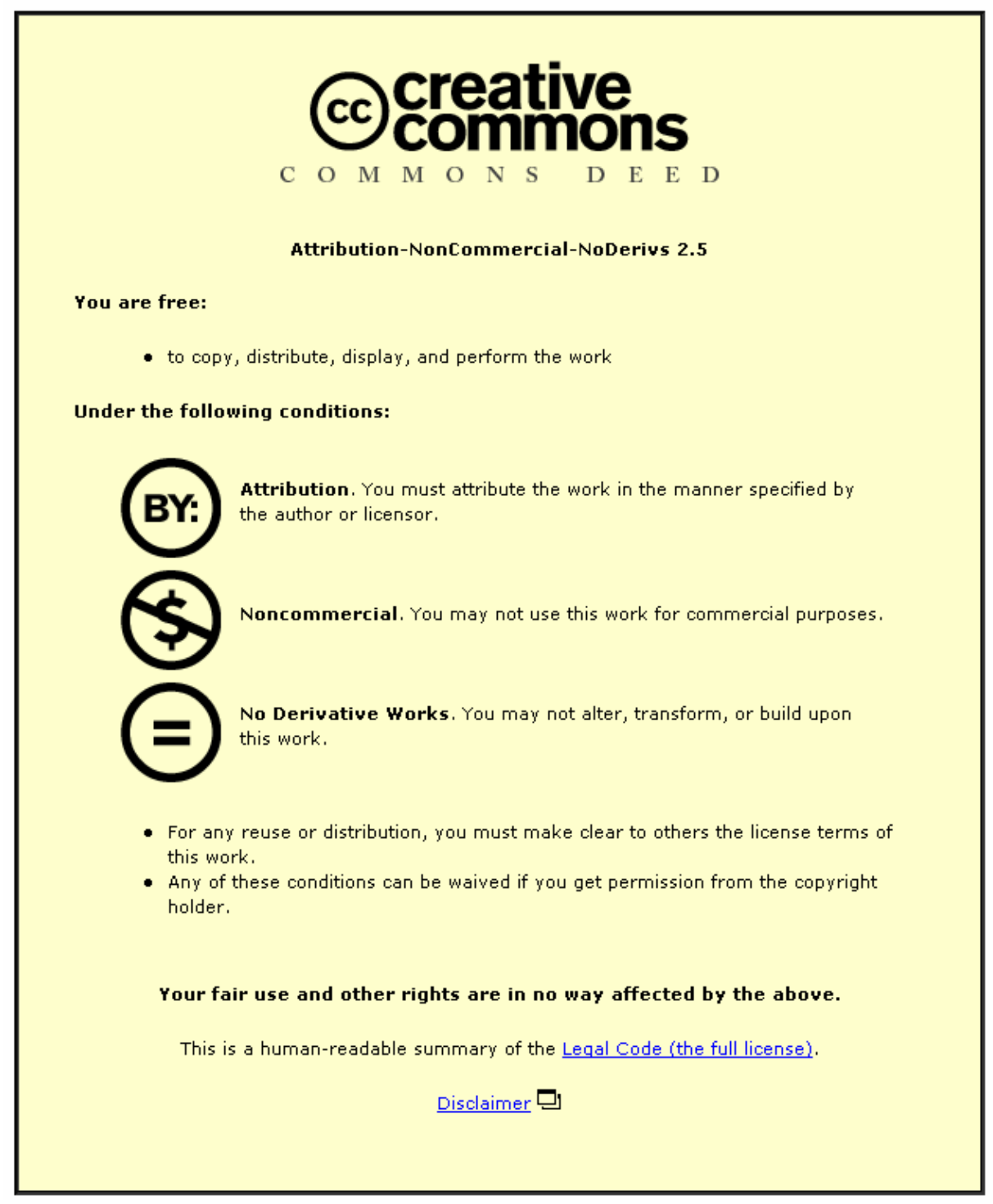

For the full text of this licence, please go to: http://creativecommons.org/licenses/by-nc-nd/2.5/ 


\title{
Testing for Seasonal Unit Roots by Frequency Domain Regression
}

\author{
Marcus J. Chambers \\ University of Essex \\ and \\ Joanne S. McGarry \\ Loughborough University
}

April 2002

\begin{abstract}
This paper considers statistics based on spectral regression estimators for testing for seasonal unit roots in a time series. An advantage of the frequency domain approach is that it enables serial correlation to be treated nonparametrically, thereby facilitating an explicit focus on the frequencies at which unit roots are of interest. The limiting distributions of the proposed test statistics are derived and their size and power properties are explored in simulation experiments.
\end{abstract}

Acknowledgements: Thanks are due to David Harvey and seminar participants at Loughborough University for helpful comments. The first author thanks the Leverhulme Trust for the financial support offered by a Philip Leverhulme Prize whilst this work was being completed.

Address for correspondence: Professor Marcus J. Chambers, Department of Economics, University of Essex, Wivenhoe Park, Colchester, Essex CO4 3SQ, England.

Tel: +44 1206 872756; fax: +44 1206 872724; e-mail: mchamb@essex.ac.uk. 


\section{INTRODUCTION}

When time series are observed on a seasonal basis, for example, quarterly or monthly, there emerges the possibility of there being unit roots at seasonal frequencies. Such unit roots are characterised in the time domain by the presence of certain roots in the autoregressive representation of the series lying on the unit circle, and in the frequency domain by poles in the spectral density at the seasonal frequencies. Most, if not all, approaches to testing for seasonal unit roots have been based upon time domain regression procedures, and many represent appropriate extensions of the methods employed for testing for a single unit root at the zero (long run) frequency. Frequency domain approaches to seasonal unit root testing appear to have been rather neglected, but, as we shall amplify below, this is not the sole reason for exploring this approach here.

As demonstrated by Phillips (1987), testing for a unit root at the zero frequency can be carried out using a straightforward regression of a variable on its lag, with any neglected (stationary) dynamics captured by the disturbance process. The limiting distribution of the resulting $t$-ratio, however, suffers from nuisance parameter dependencies that are a reflection of the dynamics in the disturbance, thus making inference difficult. One of the main approaches to this problem (among many) is to augment the regression with lagged differences in an attempt to remove the serial correlation from the disturbance (the augmented DickeyFuller approach). The latter approach has been extended to seasonal unit root testing by Hylleberg, Engle, Granger and Yoo (1990), henceforth denoted HEGY. The key ingredient from a practical point of view is to include a sufficient number of lagged (seasonal) differences as additional regressors in order to whiten the residuals, so that critical values from standard distributions (certain functionals of Wiener processes) can be used for inference. Failure to do so can result in invalid inferences being drawn. Furthermore, the outcome of such tests can be sensitive to the number of lagged differences employed.

An alternative time domain method of eradicating nuisance parameters from the limiting distributions is to make nonparametric adjustments to the test statistics, as suggested by Phillips (1987). The same goal can also be achieved by casting the regression in the frequency domain, which effectively transforms serial correlation into a type of heteroskedasticity and which can be handled by nonparametric spectral density estimators. This frequency domain regression approach was used by Choi and Phillips (1993) in testing for a unit root at the zero frequency, and we extend their methods to the case of testing for unit roots at the seasonal frequencies. One of the advantages of doing so is that it is not necessary to be concerned with selecting the appropriate number of lagged variables to include in the regression, which always takes the same form in the frequency domain. Another advantage is that the setup allows for unit root testing in autoregressive moving average (ARMA) models as well as purely autoregressive models. The key ingredient to the successful operation of the frequency domain regression approach is the ability to consistently estimate certain spectral density functions. Our simulation evidence suggests that this can be achieved straightforwardly and that the frequency domain tests are at least as powerful in finite samples as those based on a correctly specified time domain regression.

The paper is organised as follows. Section 2 defines the model and estimators, and 
obtains the limiting distributions of the proposed test statistics, which is achieved via a sequence of lemmas that provide some intermediate results. Section 3 provides simulation evidence concerning the size and power properties of the tests, assessing the impacts of different kernel functions and bandwidths in the spectral density estimation, and comparing the frequency domain tests with the corresponding tests from a correctly specified time domain regression. Section 4 concludes with some comments concerning further extensions of these methods, and two appendices provide proofs of all lemmas and theorems.

\section{THE MODEL AND TEST STATISTICS}

Consider a univariate process $w_{t}$ having the autoregressive representation

$\phi(L) w_{t}=\epsilon_{t}, \quad t=1,2, \ldots, T$,

where $\epsilon_{t} \sim \operatorname{IID}\left(0, \sigma_{\epsilon}^{2}\right), L$ denotes the lag operator, $T$ denotes sample size, and $\phi(z)=$ $1-\sum_{j=1}^{p} \phi_{j} z^{j}$ is a polynomial of order $p$, where $p \geq s$, the number of seasons. It is convenient to express the polynomial $\phi(z)$ as the product of two lower-order polynomials in the form $\phi(z)=a_{s}(z) b(z)$. In this representation $a_{s}(z)$ is a polynomial of order $s$ having $0 \leq s_{1} \leq s$ roots on the unit circle and $s-s_{1}$ roots outside the unit circle, while $b(z)$ is a polynomial of order $p-s$ that has all its roots outside the unit circle. It is, therefore, the polynomial $a_{s}(z)$ that captures possible seasonal integration in the process $w_{t}$. The issue addressed here is that of testing the number of roots of $a_{s}(z)$ that lie on the unit circle. For concreteness we focus on the case $s=4$, which is appropriate for testing for seasonal unit roots with quarterly data. The general methods, however, can be straightforwardly extended to other values of $s$ as required.

With the factorisation of $\phi(z)$ described above and $s=4$ it is possible to write (1) as

$a_{4}(L) w_{t}=u_{t}$,

where $u_{t}=c(L) \epsilon_{t}$ is a stationary random disturbance in which $c(z)=1+\sum_{j=1}^{\infty} c_{j} z^{j}=b(z)^{-1}$. Following Hylleberg, Engle, Granger and Yoo (1990), the polynomial $a_{4}(z)=1+\sum_{j=1}^{4} a_{j} z^{j}$ can be decomposed as

$a_{4}(z)=-\beta_{1} z \alpha_{1}(z)-\beta_{2} z \alpha_{2}(z)-\beta_{3} z \alpha_{3}(z)-\beta_{4} z \alpha_{4}(z)+\left(1-z^{4}\right)$,

where $\alpha_{1}(z)=1+z+z^{2}+z^{3}, \alpha_{2}(z)=-\left(1-z+z^{2}-z^{3}\right), \alpha_{3}(z)=-z\left(1-z^{2}\right)$ and $\alpha_{4}(z)=-\left(1-z^{2}\right)$. The coefficients $\beta_{1}, \ldots, \beta_{4}$ correspond to the roots $1,-1, i=\sqrt{-1}$ and $-i$ respectively in the sense that if $\beta_{j}=0$ then $a_{4}(z)$ possesses the corresponding root. Matching the coefficients in (3) with those of $a_{4}(z)$ yields the relationships $a_{1}=-\beta_{1}+\beta_{2}+\beta_{4}$, $a_{2}=-\beta_{1}-\beta_{2}+\beta_{3}, a_{3}=-\beta_{1}+\beta_{2}-\beta_{4}$, and $a_{4}=-\beta_{1}-\beta_{2}-\beta_{3}-1$. Alternatively, solving these expressions for the $\beta_{j}$ in terms of the $a_{j}$ yields $\beta_{1}=-a_{4}(1) / 4, \beta_{2}=-\left(1-a_{1}+a_{2}-a_{3}+a_{4}\right) / 4$, $\beta_{3}=-\left(1-a_{2}+a_{4}\right) / 2$ and $\beta_{4}=\left(a_{1}-a_{3}\right) / 2$. The point to note about the representation (3) is that the term $\left(1-z^{4}\right)$ is not multiplied by another polynomial, as in Hylleberg, Engle, 
Granger and Yoo (1990), in which a representation of $\phi(z)$, rather than just $a_{4}(z)$, is sought and which results in a term of the form $a^{*}(z)\left(1-z^{4}\right)$, where $a^{*}(z)$ is a finite polynomial. This term is required by HEGY to account for the additional terms in the polynomial involving $b(z)$, which in our approach has already been incorporated within the disturbance term $u_{t}$.

Let $y_{t}=\left(1-L^{4}\right) w_{t}$ and define the variables $y_{j t}=\alpha_{j}(L) w_{t}(j=1, \ldots, 4)$. Then, using the representation (3) for $a_{4}(z)$, (2) may be written as the regression model

$y_{t}=\sum_{j=1}^{4} \beta_{j} y_{j, t-1}+u_{t}, \quad t=1,2, \ldots, T$

which is in the form of the HEGY regression ${ }^{1}$ but with one notable exception. In the HEGY approach, the aim is to produce a white noise disturbance, and hence their representation of $\phi(z)$ yields a variable on the left hand side which may be denoted $a^{*}(L) y_{t}$ and which incorporates the dynamics that are effectively associated with $b(z)$. In the spectral regression approach adopted here such dynamics are assigned to the disturbance term $u_{t}$ and are treated nonparametrically via the use of appropriate spectral density estimates.

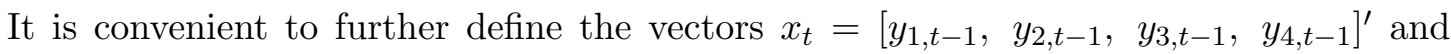
$\beta=\left[\beta_{1}, \beta_{2}, \beta_{3}, \beta_{4}\right]^{\prime}$. Then (4) may be written as the regression model

$y_{t}=x_{t}^{\prime} \beta+u_{t}, \quad t=1,2, \ldots, T$.

The frequency domain tests of the restrictions $\beta_{j}=0(j=1, \ldots, 4)$ considered here are based on the frequency domain regression estimator of $\beta$ defined by

$\hat{\beta}=\left[\frac{1}{2 M} \sum_{j=-M+1}^{M} \hat{f}_{x x}\left(\omega_{j}\right) \hat{f}_{\hat{u} \hat{u}}\left(\omega_{j}\right)^{-1}\right]^{-1}\left[\frac{1}{2 M} \sum_{j=-M+1}^{M} \hat{f}_{x y}\left(\omega_{j}\right) \hat{f}_{\hat{u} \hat{u}}\left(\omega_{j}\right)^{-1}\right]$.

In the above definition of $\hat{\beta}, \hat{f}_{a b}(\omega)$ denotes a nonparametric estimate of the spectral density function of two (possibly vector) random processes $a_{t}$ and $b_{t}$, given by

$\hat{f}_{a b}(\omega)=\frac{1}{2 \pi} \sum_{n=-M}^{M} k\left(\frac{n}{M}\right) C_{a b}(n) e^{-i n \omega}$,

where $k(\cdot)$ is a kernel function (or lag window), $M$ is a bandwidth parameter, $\omega_{j}=\pi j / M$, and

$C_{a b}(n)= \begin{cases}T^{-1} \sum_{t=1}^{T-n} a_{t} b_{t+n}^{\prime}, & n \geq 0, \\ T^{-1} \sum_{t=|n|+1}^{T} a_{t} b_{t-|n|}^{\prime}, & n<0 .\end{cases}$

\footnotetext{
${ }^{1}$ The variable $y_{4 t}$ is denoted $y_{3 t}$ by HEGY and $y_{3 t}$ is HEGY's $y_{3, t-1}$.
} 
The variable $\hat{u}_{t}=y_{t}-x_{t}^{\prime} \hat{\beta}_{O L S}$ denotes the residual from a time domain regression of $y_{t}$ on $x_{t}$, where $\hat{\beta}_{O L S}$ is the ordinary least squares estimator. The estimated asymptotic covariance matrix of $\hat{\beta}$ is

$V_{T}=\frac{1}{T}\left[\frac{1}{2 M} \sum_{j=-M+1}^{M} \hat{f}_{x x}\left(\omega_{j}\right) \hat{f}_{\hat{u} \hat{u}}\left(\omega_{j}\right)^{-1}\right]^{-1}$.

Two types of test statistic are considered for testing the individual null hypotheses that $\beta_{j}=0(j=1, \ldots, 4)$. The first type of statistic is simply $T \hat{\beta}_{j}(j=1, \ldots, 4)$ while the second type is the t-ratio defined by

$t_{j}=\frac{\hat{\beta}_{j}}{V_{T, j j}^{1 / 2}}, \quad j=1, \ldots, 4$,

where $V_{T, j j}$ denotes the $j$ 'th diagonal element of $V_{T}$. Certain hypotheses concerning the joint significance of the $\beta_{j}$ coefficients are also of interest. Here we shall focus on two of these, namely that $\beta_{3}=\beta_{4}=0$ and that $\beta_{1}=\beta_{2}=\beta_{3}=\beta_{4}=0$. These hypotheses can be tested using Wald statistics constructed from the unrestricted estimator $\hat{\beta}$. The Wald statistics will be denoted $J_{34}$ and $J_{1234}$ respectively, and are defined by

$J_{i}=\hat{\beta}^{\prime} R_{i}^{\prime}\left[R_{i} V_{T} R_{i}^{\prime}\right]^{-1} R_{i} \hat{\beta}, \quad i=1234,34$,

where $R_{1234}=I_{4}, R_{34}=\left[0_{2}, I_{2}\right], 0_{n}$ denotes an $n \times n$ matrix of zeros, $I_{n}$ denotes an $n \times n$ identity matrix, and $V_{T}$ is the covariance matrix defined in (9). The limiting distributions of these statistics are derived under the following assumption concerning the process $u_{t}$.

Assumption 1. The process $u_{t}$ in (2) is given by $u_{t}=c(L) \epsilon_{t}$ where $c(z)=1+\sum_{j=1}^{\infty} c_{j} z^{j}$ satisfies $\sum_{j=1}^{\infty} j^{2} c_{j}^{2}<\infty$ and $\epsilon_{t} \sim \operatorname{IID}\left(0, \sigma_{\epsilon}^{2}\right)$ with $\sigma_{\epsilon}^{2}<\infty$.

Assumption 1 ensures, in particular, that certain partial sums of $u_{t}$ satisfy an invariance principle. Assumption 1 is clearly satisfied by $u_{t}$ as defined earlier provided $c(z)=b(z)^{-1}$ satisfies the appropriate summability condition for its coefficients. However, it also allows for circumstances in which $\epsilon_{t}$ in (1) is not IID but is itself a linear process. The partial sums of interest are

$P_{t}=\sum_{j=1}^{t} u_{j}, \quad M_{t}=\sum_{j=1}^{t}(-1)^{j} u_{j}, \quad C_{t}=\sum_{j=1}^{t} \cos \frac{\pi j}{2} u_{j} \quad$ and $\quad S_{t}=\sum_{j=1}^{t} \sin \frac{\pi j}{2} u_{j}$

because under the null hypothesis that $\left(1-L^{4}\right) w_{t}=u_{t}$, the variables $y_{j t}$ can be expressed in terms of these partial sums as follows:

$y_{1 t}=(1-L)^{-1} u_{t}=\sum_{j=1}^{t} u_{j}=P_{t}$ 


$$
\begin{aligned}
& y_{2 t}=-(1+L)^{-1} u_{t}=-(-1)^{t} \sum_{j=1}^{t}(-1)^{j} u_{j}=-(-1)^{t} M_{t}, \\
& y_{4 t}=-\left(1+L^{2}\right)^{-1} u_{t}=-\sum_{k=1}^{t} \sin \left[\frac{(t-k+1) \pi}{2}\right] u_{k}=S_{t} \cos \left[\frac{(t+1) \pi}{2}\right]-C_{t} \sin \left[\frac{(t+1) \pi}{2}\right],
\end{aligned}
$$

the last line using the identity $\sin (x-y)=\sin x \cos y-\cos x \sin y$ with $x=(t+1) \pi / 2$ and $y=k \pi / 2$. The following invariance principles hold under Assumption 1.

LEMMA 1. Under Assumption 1, as $T \rightarrow \infty$ :

(a) $T^{-\frac{1}{2}} P_{[T r]}=T^{-\frac{1}{2}} \sum_{j=1}^{[T r]} u_{j} \Rightarrow \sigma_{\epsilon} c(1) W_{1}(r)$,

(b) $T^{-\frac{1}{2}} M_{[T r]}=T^{-\frac{1}{2}} \sum_{j=1}^{[T r]}(-1)^{j} u_{j} \Rightarrow \sigma_{\epsilon} c(-1) W_{2}(r)$,

(c) $T^{-\frac{1}{2}}\left[C_{[T r]} \pm i S_{[T r]}\right]=T^{-\frac{1}{2}} \sum_{j=1}^{[T r]} e^{ \pm i \pi j / 2} u_{j} \Rightarrow \frac{\sigma_{\epsilon}}{\sqrt{2}} c\left(e^{ \pm i \pi / 2}\right)\left[W_{3}(r) \pm i W_{4}(r)\right]$,

where the $W_{j}(j=1, \ldots, 4)$ are independent Wiener processes on $r \in[0,1]$ and $[\mathrm{Tr}]$ denotes the integer part of $\mathrm{Tr}$.

Letting $c_{R}$ and $c_{I}$ denote the real and imaginary parts of $c\left(e^{ \pm i \pi / 2}\right)$ respectively, an immediate implication of Lemma $1(\mathrm{c})$ is that

$$
\begin{aligned}
& T^{-\frac{1}{2}} C_{[T r]}=T^{-\frac{1}{2}} \sum_{j=1}^{[T r]} \cos (\pi j / 2) u_{j} \Rightarrow \frac{\sigma_{\epsilon}}{\sqrt{2}}\left[c_{R} W_{3}(r)-c_{I} W_{4}(r)\right], \\
& T^{-\frac{1}{2}} S_{[T r]}=T^{-\frac{1}{2}} \sum_{j=1}^{[T r]} \sin (\pi j / 2) u_{j} \Rightarrow \frac{\sigma_{\epsilon}}{\sqrt{2}}\left[c_{I} W_{3}(r)+c_{R} W_{4}(r)\right] .
\end{aligned}
$$

The limiting distributions of the test statistics are derived under the assumption that all the $\beta_{j}$ coefficients are equal to zero, in which case $\left(1-L^{4}\right) w_{t}=u_{t}$. In this situation, the individual null hypotheses would be interpreted as $\beta_{j}=0$ given the other $\beta_{k}(k \neq j)$ are zero. It will be shown later, however, that the forms of limiting distributions are unaltered even if the other $\beta_{k} \neq 0$. The derivations of the asymptotic properties require a description of the limiting behaviour of the spectral density estimates of the form (7), which in turn are based on the sample autocovariance estimates in (8). The following lemma depicts the appropriate asymptotics for the relevant sample autocovariances. 
LEMMA 2. Under Assumption 1 and assuming that $\left(1-L^{4}\right) w_{t}=u_{t}$, the following sample moments converge to the stated limits as $T \rightarrow \infty$ :

(a) $\frac{1}{T^{2}} \sum_{t=1}^{T-n} y_{1 t} y_{1, t+n} \Rightarrow \sigma_{\epsilon}^{2} c(1)^{2} \int_{0}^{1} W_{1}^{2}$,

(b) $\frac{1}{T^{2}} \sum_{t=1}^{T-n} y_{2 t} y_{2, t+n} \Rightarrow(-1)^{n} \sigma_{\epsilon}^{2} c(-1)^{2} \int_{0}^{1} W_{2}^{2}$,

(c) $\frac{1}{T^{2}} \sum_{t=1}^{T-n} y_{4 t} y_{4, t+n} \Rightarrow\left\{\begin{array}{l}(-1)^{\frac{n}{2}} \frac{\sigma_{\epsilon}^{2}}{4}\left|c\left(e^{i \pi / 2}\right)\right|^{2} \int_{0}^{1}\left[W_{3}^{2}+W_{4}^{2}\right], \quad n \text { even } \\ 0, \quad n \text { odd }\end{array}\right.$

(d) $\frac{1}{T^{2}} \sum_{t=1}^{T-n} y_{1 t} y_{j, t+n} \stackrel{p}{\rightarrow} 0, \quad j=2,4$,

(e) $\frac{1}{T^{2}} \sum_{t=1}^{T-n} y_{2 t} y_{j, t+n} \stackrel{p}{\rightarrow} 0, \quad j=1,4$,

where $\int_{0}^{1} W_{i}^{2}(i=1, \ldots, 4)$ denotes the integral $\int_{0}^{1} W_{i}(r)^{2} d r$.

Note that, since $y_{3 t}=y_{4, t-1}$, the Lemma also implicitly provides the appropriate results for the sample autocovariances involving $y_{3 t}$. In particular, the Lemma implies that $T^{-2} \sum_{t=1}^{T-n} y_{4 t} y_{3, t+n}$ converges to a limiting functional of Wiener processes for $n$ odd, given by the result in Lemma 2(c) for $n$ even. In addition to the above results for the convergence of sample moments, it is also necessary to derive results for terms of the form $T^{-1} \sum_{t=1}^{T-n} y_{j, t-1} u_{t+n}$ that arise in the analysis of the spectral regression estimator. A key step in achieving this is the limiting distributions given in Lemma 3.

LEMMA 3. Under Assumption 1, as $T \rightarrow \infty$ :

(a) $\frac{1}{T} \sum_{t=1}^{T} P_{t-1} u_{t} \Rightarrow \sigma_{\epsilon}^{2} c(1)^{2} \int_{0}^{1} W_{1} d W_{1}+\delta_{1}(1)$,

(b) $\frac{1}{T} \sum_{t=1}^{T} M_{t-1}(-1)^{t} u_{t} \Rightarrow \sigma_{\epsilon}^{2} c(-1)^{2} \int_{0}^{1} W_{2} d W_{2}+\delta_{2}(1)$,

(c) $\frac{1}{T} \sum_{t=1}^{T}\left(C_{t-1} \cos \frac{\pi t}{2} u_{t}+S_{t-1} \sin \frac{\pi t}{2} u_{t}\right) \Rightarrow \frac{\sigma_{\epsilon}^{2}}{2}\left|c\left(e^{i \pi / 2}\right)\right|^{2} \int_{0}^{1}\left[W_{3} d W_{3}+W_{4} d W_{4}\right]+\delta_{3}(1)$,

(d) $\frac{1}{T} \sum_{t=1}^{T}\left(C_{t-1} \sin \frac{\pi t}{2} u_{t}-S_{t-1} \cos \frac{\pi t}{2} u_{t}\right) \Rightarrow \frac{\sigma_{\epsilon}^{2}}{2}\left|c\left(e^{i \pi / 2}\right)\right|^{2} \int_{0}^{1}\left[W_{3} d W_{4}-W_{4} d W_{3}\right]+\delta_{4}(0)$,

where $\int_{0}^{1} W_{i} d W_{j}$ denotes the stochastic integral $\int_{0}^{1} W_{i}(r) d W_{j}(r)$ and, defining $\gamma_{j}=E\left(u_{0} u_{j}\right)$, $\delta_{1}(n)=\sum_{j=n}^{\infty} \gamma_{j}, \quad \delta_{2}(n)=\sum_{j=n}^{\infty}(-1)^{j} \gamma_{j}, \quad \delta_{3}(n)=\sum_{j=n}^{\infty}(-1)^{j} \gamma_{2 j}, \quad$ and $\quad \delta_{4}(n)=\sum_{j=n}^{\infty}(-1)^{j} \gamma_{2 j+1}$. 
The results in Lemma 3 can be used to derive the limiting properties of moments of the form $\sum_{t=1}^{T-n} y_{j, t-1} u_{t+n}(j=1, \ldots, 4)$. These are presented in Lemma 4 .

LEMMA 4. Under Assumption 1 and assuming that $\left(1-L^{4}\right) w_{t}=u_{t}$, as $T \rightarrow \infty$ :

(a) $\frac{1}{T} \sum_{t=1}^{T-n} y_{1, t-1} u_{t+n} \Rightarrow \sigma_{\epsilon}^{2} c(1)^{2} \int_{0}^{1} W_{1} d W_{1}+\delta_{1}(n+1)$,

(b) $\frac{1}{T} \sum_{t=1}^{T-n} y_{2, t-1} u_{t+n} \Rightarrow(-1)^{n}\left\{\sigma_{\epsilon}^{2} c(-1)^{2} \int_{0}^{1} W_{2} d W_{2}+\delta_{2}(n+1)\right\}$,

(c) $\frac{1}{T} \sum_{t=1}^{T-n} y_{4, t-1} u_{t+n} \Rightarrow\left\{\begin{array}{r}(-1)^{\frac{(n+2)}{2}}\left[\frac{\sigma_{\epsilon}^{2}}{2}\left|c\left(e^{i \pi / 2}\right)\right|^{2} \int_{0}^{1}\left(W_{3} d W_{4}-W_{4} d W_{3}\right)+\delta_{4}(n)\right], \\ n \text { even }, \\ (-1)^{\frac{(n-1)}{2}}\left[\frac{\sigma_{\epsilon}^{2}}{2}\left|c\left(e^{i \pi / 2}\right)\right|^{2} \int_{0}^{1}\left(W_{3} d W_{3}+W_{4} d W_{4}\right)+\delta_{3}(n)\right], \\ n \text { odd },\end{array}\right.$

where $\delta_{1}(n), \delta_{2}(n), \delta_{3}(n)$ and $\delta_{4}(n)$ are defined in Lemma 3.

Once again, since $y_{3 t}=y_{4, t-1}$, Lemma 4 also implicitly provides the results for terms involving $y_{3, t-1}$. The results in Lemma 4 , allied to those in Lemma 2, are utilised in deriving the asymptotic properties of the estimator $\hat{\beta}$, which also requires the following assumption.

Assumption 2. The lag window $k(x)$ is a bounded, even, function satisfying $k(0)=1$ and $k(x)=0$ for $x \notin[-1,1]$. As $T \rightarrow \infty$, the bandwidth parameter $M \rightarrow \infty$ but in a way such that $M / T^{\frac{1}{2}} \rightarrow 0$

The restrictions on $k(\cdot)$ and $M$ imposed by Assumption 2 are common in the literature; see, for example, Hannan (1970) and Choi and Phillips (1993).

THEOREM 1. Under Assumptions 1 and 2 and assuming that $\left(1-L^{4}\right) w_{t}=u_{t}$,

$\frac{1}{2 M T} \sum_{j=-M+1}^{M} \hat{f}_{x x}\left(\omega_{j}\right) f_{u u}\left(\omega_{j}\right)^{-1} \Rightarrow \operatorname{diag}\left\{H_{k k}\right\} \quad(k=1, \ldots, 4)$,

$\frac{1}{2 M} \sum_{j=-M+1}^{M} \hat{f}_{x u}\left(\omega_{j}\right) f_{u u}\left(\omega_{j}\right)^{-1} \Rightarrow\left[h_{1}, h_{2}, h_{3}, h_{4}\right]^{\prime}$, 
as $T \rightarrow \infty$, where

$$
\begin{aligned}
& H_{11}=\int_{0}^{1} W_{1}^{2}, \quad H_{22}=\int_{0}^{1} W_{2}^{2}, \quad H_{33}=H_{44}=\frac{1}{4} \int_{0}^{1}\left(W_{3}^{2}+W_{4}^{2}\right), \\
& h_{1}=\int_{0}^{1} W_{1} d W_{1}, \quad h_{2}=\int_{0}^{1} W_{2} d W_{2}, \\
& h_{3}=\frac{1}{2} \int_{0}^{1}\left(W_{3} d W_{3}+W_{4} d W_{4}\right), \quad h_{4}=\frac{1}{2} \int_{0}^{1}\left(W_{3} d W_{4}-W_{4} d W_{3}\right) .
\end{aligned}
$$

Theorem 1 enables, in particular, the distribution of $T(\hat{\beta}-\beta)$ to be derived. A key feature of the results in Theorem 1 is that the limiting distributions are free of nuisance parameter dependencies that may otherwise arise due to the serial correlation in $u_{t}$. In effect these nuisance parameters are eradicated by the frequency domain estimator of $\beta$ in (5) in a way not achieved by the OLS estimator, even though the frequency domain estimator utilises the OLS residuals in constructing the spectral density estimates. The asymptotic properties of the test statistics of interest are given in Theorem 2 .

THEOREM 2. Under Assumptions 1 and 2 and assuming that $\left(1-L^{4}\right) w_{t}=u_{t}$, the limiting distributions as $T \rightarrow \infty$ of the test statistics are given by:

$$
\begin{array}{ll}
T \hat{\beta}_{1} \Rightarrow \frac{\int_{0}^{1} W_{1} d W_{1}}{\int_{0}^{1} W_{1}^{2}}, & t_{1} \Rightarrow \tau_{1}=\frac{\int_{0}^{1} W_{1} d W_{1}}{\left[\int_{0}^{1} W_{1}^{2}\right]^{\frac{1}{2}}} \\
T \hat{\beta}_{2} \Rightarrow \frac{\int_{0}^{1} W_{2} d W_{2}}{\int_{0}^{1} W_{2}^{2}}, & t_{2} \Rightarrow \tau_{2}=\frac{\int_{0}^{1} W_{2} d W_{2}}{\left[\int_{0}^{1} W_{2}^{2}\right]^{\frac{1}{2}}} \\
T \hat{\beta}_{3} \Rightarrow \frac{2 \int_{0}^{1}\left(W_{3} d W_{3}+W_{4} d W_{4}\right)}{\int_{0}^{1}\left(W_{3}^{2}+W_{4}^{2}\right)}, \quad t_{3} \Rightarrow \tau_{3}=\frac{\int_{0}^{1}\left(W_{3} d W_{3}+W_{4} d W_{4}\right)}{\left[\int_{0}^{1}\left(W_{3}^{2}+W_{4}^{2}\right)\right]^{\frac{1}{2}}} \\
T \hat{\beta}_{4} \Rightarrow \frac{2 \int_{0}^{1}\left(W_{4} d W_{3}-W_{3} d W_{4}\right)}{\int_{0}^{1}\left(W_{3}^{2}+W_{4}^{2}\right)}, \quad t_{4} \Rightarrow \tau_{4}=\frac{\int_{0}^{1}\left(W_{4} d W_{3}-W_{3} d W_{4}\right)}{\left[\int_{0}^{1}\left(W_{3}^{2}+W_{4}^{2}\right)\right]^{\frac{1}{2}}} \\
J_{34} \Rightarrow \tau_{3}^{2}+\tau_{4}^{2},
\end{array}
$$

The limiting distributions given in Theorem 2 are familiar in the literature on seasonal unit root tests and seasonal cointegration. Variants can be found, for example, in Engle, Granger, 
Hylleberg and Lee (1993), Ghysels, Lee and Noh (1994) and Smith and Taylor (1998). The key feature of Theorem 2 for the purposes of inference is that the limiting distributions are free of nuisance parameters. Each distribution is expressed entirely in terms of standard Wiener processes. This 'optimality' result is achieved here via the use of nonparametric spectral density estimates to account for nuisance parameters that would otherwise arise due to the serial correlation inherent in $u_{t}$. Similar results arise in the time domain HEGY regressions because such serial correlation is accounted for by incorporating lagged $\left(1-L^{4}\right) w_{t}$ terms as additional regressors so that the disturbance term in the regression is $\epsilon_{t}$. In the time domain the validity of these asymptotic distributions boils down to the ability to correctly specify the order of the polynomial $\phi(z)$ in (1). In the frequency domain the key feature is to be able to consistently estimate certain spectral densities.

The limiting distributions presented in Theorem 2 have been derived under the null hypothesis that $\left(1-L^{4}\right) w_{t}=u_{t}$, so that the tests of individual restrictions are to be interpreted as tests of the hypothesis that $\beta_{j}=0$ given that all other $\beta_{k}=0(k \neq j)$. The same distributions hold, though, even if the other $\beta_{k} \neq 0$.

COROLLARY TO THEOREM 2. The limiting distributions of the tests statistics $T \hat{\beta}_{j}$ and $t_{j}(j=1, \ldots, 4)$ remain valid even if $\beta_{k} \neq 0(k \neq j)$.

The tests of individual restrictions in Theorem 2 therefore hold regardless of whether there are unit roots at other frequencies or not.

\section{EXPERIMENTAL EVIDENCE}

The finite sample performance of the frequency domain test statistics can be assessed by means of appropriately-designed simulations. The simulation results reported in this section are aimed at addressing the following issues. The main focus is the size and power properties of the tests. Many tests for unit roots have been found to suffer from potentially severe size distortions and from lack of power, so this issue is also important in the context of seasonal unit roots. Secondly, it is of interest to investigate how the properties of the tests are affected by varying the bandwidth parameter $M$ and the lag window $k(\cdot)$. Finally, a comparison of the properties of the frequency domain tests with the time domain HEGY tests also seems pertinent.

The model used to generate the simulated data to assess the size properties of the tests is given by

$(1+\psi L)\left(1-L^{4}\right) w_{t}=\epsilon_{t}, \quad \epsilon_{t} \sim \operatorname{NID}(0,1)$,

where $\psi \in\{-0.5,-0.2,0,0.2,0.5\}$. In terms of $(1), \phi(z)=1+\psi z-z^{4}-\psi z^{5}$, while in terms of $(2), a_{4}(z)=1-z^{4}$ and $c(z)=(1+\psi z)^{-1}=\sum_{j=0}^{\infty}(-1)^{j} \psi^{j} z^{j}$. The variable $w_{t}$ therefore has unit roots at all four seasonal frequencies. A total of 10,000 replications were carried out 
with sample sizes $T=48,100,200$ corresponding to 12,25 and 50 years of quarterly data respectively. The HEGY regression corresponding to (12) is

$\left(1-L^{4}\right) w_{t}=\sum_{j=1}^{4} \beta_{j} y_{j, t-1}-\psi\left(1-L^{4}\right) w_{t-1}+\epsilon_{t}$,

where the $y_{j t}$ are defined prior to (4). Note that the HEGY regression is correctly specified in these simulations, a situation that is not guaranteed in practise with real data. In addition to the $t$-tests of the individual hypotheses that $\beta_{j}=0$, the HEGY regression is also used to produce $F$-statistics for the hypotheses $\beta_{1}=\beta_{2}=\beta_{3}=\beta_{4}=0$ and $\beta_{3}=\beta_{4}=0$. These statistics are denoted $F_{1234}$ and $F_{34}$ respectively. Critical values for the frequency domain tests were obtained from an initial set of 40,000 replications with $\psi=0 .^{2}$ Critical values for the HEGY $t_{i}$ and $F_{34}$ tests were obtained from Hylleberg, Engle, Granger and Yoo (1990), while those for the $F_{1234}$ test were obtained from Ghysels, Lee and Noh (1994). Note that the normalised bias test statistics $T \hat{\beta}_{i}$ are not usually carried out in the HEGY framework and so we do not consider them here either. The power of the tests was assessed by generating data according to

$(1+\psi L)\left(1-0.85 L^{4}\right) w_{t}=\epsilon_{t}, \quad \epsilon_{t} \sim \operatorname{NID}(0,1)$,

in which the roots are \pm 1.0415 and $\pm 1.0415 i$.

In order to assess the effects of the choice of bandwidth and kernel function on the tests, two kernels and two bandwidth values were used. The kernel functions were the Parzen kernel, defined by

$k(x)= \begin{cases}1-6 x^{2}+6|x|^{3}, & |x| \leq 0.5, \\ 2(1-|x|)^{3}, & 0.5<|x| \leq 1, \\ 0, & |x|>1,\end{cases}$

and the Tukey kernel (also known as the Tukey-Hanning kernel), given by

$k(x)= \begin{cases}0.5(1+\cos \pi x), & |x| \leq 1 \\ 0, & |x|>1 .\end{cases}$

The bandwidth parameter was chosen to satisfy $M=\left[T^{\delta}\right]+1$ for $\delta=\{1 / 5,1 / 3\}$. Xiao and Phillips (1998) demonstrated that the optimal bandwidth for the estimators considered here is $O\left(T^{1 / 3}\right)$, while that for an alternative spectral estimator is $O\left(T^{1 / 5}\right)$. Optimality in this context refers to the value of $M$ that minimises the asymptotic mean square error of the estimator, and so is not directly related to optimal test performance, hence we consider both values in our simulations.

[Tables 1, 2 and 3 about here.]

\footnotetext{
${ }^{2}$ Critical values can be obtained from the authors on request.
} 
The size properties of the tests are reported in Tables 1, 2 and 3. Table 1 contains the results for the frequency domain tests based on the Tukey kernel, Table 2 the results based on the Parzen kernel, and Table 3 the results for the time domain (HEGY) tests. In all cases the nominal size of the tests is 0.05 . The empirical size of the frequency domain statistics is close to the nominal size when $\psi=0$ but size distortions are apparent when $\psi \neq 0$, although these distortions tend to reduce as sample size increases. This is partly to be expected because the critical values were obtained for the case $\psi=0$ and, asymptotically, the distributions are invariant to the value of $\psi$. Such size distortions are also evident for the frequency domain unit root tests in Choi and Phillips (1993). Another possible reason for the size distortions can be gleaned from Figure 1, which plots the spectral density of $u$ for the different values of $\psi$ used in the simulations. The spectrum is flat when $\psi=0$ but contains a peak at frequency $\pi$ and a trough at the zero frequency when $\psi>0$ while the reverse is true when $\psi<0$. These peaks and troughs are more difficult to estimate than a purely flat spectrum, although critical values drawn from experiments using the correct value of $\psi$ would obviously eradicate the size distortions. It is interesting to note that the empirical size of the time domain tests is close to the nominal value even for $\psi \neq 0$.

[Figure 1 and Tables 4, 5 and 6 about here.]

The power of the tests is reported in Tables 4, 5 and 6 , the first two for the frequency domain tests, the third for the time domain tests. On the whole, the frequency domain tests based on the Parzen kernel appear to have higher power than those based on the Tukey kernel, although the differences are fairly small and are not in an entirely uniform direction. Also there are differences in the power performance between different values of the bandwidth parameter, which is again not uniform and in some circumstances the tests based on $\delta=1 / 5$ have higher power than those based on the optimal value $\delta=1 / 3$. There are also notable differences in the power of the tests according to the value of the parameter $\psi$. The power of the frequency domain tests of $\beta_{1}=0$ declines with $\psi$ while the reverse is true of tests of $\beta_{2}=0$. A partial explanation of this can be found by examining the spectral density function of $w_{t}$ under the alternative hypothesis. Figure 2 depicts the logarithm of the spectral density of $w$ for each value of $\psi$ considered in the experiments. As can be seen, when $\psi>0$, the frequency $\pi$ corresponds to a sharp peak in the spectrum of $w$, which is difficult to distinguish from a pole at this frequency, which would correspond to a unit root. This means that it is harder for the tests to reject the (false) null hypothesis that $\beta_{2}=0$ which explains the low power in this scenario. As $\psi$ decreases in value and becomes negative, the peak at frequency $\pi$ becomes less accentuated and the corresponding expected increase in power is borne out in the simulations. The reverse is true at the zero frequency, with the peak more accentuated when $\psi<0$, which explains the increase in the power of the test of the null that $\beta_{1}=0$ when $\psi$ becomes positive.

[Figure 2 about here.]

A striking feature of the results in Tables 4, 5 and 6 is that the power of the frequency domain tests is, in many instances, at least as high as the time domain tests, even at the 
smallest sample size $(T=48)$, although a uniform ranking does not emerge. The performance of the tests of the joint hypotheses is particularly noteworthy. This is encouraging for the use of frequency domain tests in practice, because they are based on a nonparametric treatment of dynamics whereas, in the simulations, the time domain regression is correctly specified. This suggests that the frequency domain nonparametric corrections work rather well even when compared with correctly parametrised alternatives in the time domain.

\section{CONCLUDING COMMENTS}

In addition to deriving the asymptotic properties of frequency domain regression based tests of seasonal unit roots, it has been demonstrated in simulations that the tests can perform well in finite samples when compared to time domain regression based tests. This is quite striking because the frequency domain tests rely on nonparametric spectral density estimates to account for the serial correlation in the disturbances, whereas the time domain regressions are correctly specified. Applications, and further comparisons, using real data are being pursued by the authors.

There are many ways in which the methods can be extended. The model considered here contains no deterministic terms, but it would be straightforward to include a constant, time trend and seasonal dummies if required. The easiest way to deal with such deterministic terms is to detrend the data by a time domain regression prior to the regression in the frequency domain. The limiting distributions would then be characterised by suitably detrended Wiener processes. Such detrending is a valid procedure because the parameters to be estimated in the frequency domain regression are independent of frequency, although it would be of interest to also consider frequency domain detrending, along the lines detailed in Corbae, Ouliaris and Phillips (2002) who show that time domain detrending is not efficient if the regression of interest contains frequency-dependent parameters. Frequency domain regression could also be employed in the estimation of parameters in seasonally cointegrated models, and the results derived here could provide a basis for such a study. 


\section{REFERENCES}

Chan, N. H. and Wei, C. Z. (1988) Limiting distributions of least squares estimates of unstable autoregressive processes. Annals of Statistics 16, 367-401.

Choi, I. and Phillips, P. C. B. (1993) Testing for a unit root by frequency domain regression. Journal of Econometrics 59, 263-286.

Corbae, D., Ouliaris, S. and Phillips, P. C. B. (2002) Band spectral regression with trending data. Econometrica 70, (forthcoming).

Engle, R. F., Granger, C. W. J., Hylleberg, S. and Lee, H. S. (1993) Seasonal cointegration: the Japanese consumption function. Journal of Econometrics 55, 275-298.

Ghysels, E., Lee, H. S. and Noh, J. (1994) Testing for unit roots in seasonal time series. Journal of Econometrics 62, 415-442.

Gradshteyn, I. S. and Ryzhik, I. M. (1994) Table of Integrals, Series, and Products. Academic Press, New York, 5th edition.

Hannan, E. J. (1963) Regression for time series. In M. Rosenblatt (ed.), Time Series Analysis, chapter 2, pp. 17-37. Wiley, New York.

Hannan, E. J. (1970) Multiple Time Series. Wiley, New York.

Hylleberg, S., Engle, R. F., Granger, C. W. J. and Yoo, B. S. (1990) Seasonal integration and cointegration. Journal of Econometrics 44, 215-238.

Phillips, P. C. B. (1987) Time series regression with a unit root. Econometrica 55, 277-301.

Phillips, P. C. B. (1988) Weak convergence of sample covariance matrices to stochastic integrals via martingale approximations. Econometric Theory 4, 528-533.

Phillips, P. C. B. (1991) Spectral regression for cointegrated time series. In W. A. Barnett, J. Powell and G. Tauchen (eds.), Nonparametric and Semiparametric Methods in Economics and Statistics, chapter 16, pp. 413-435. Cambridge University Press, Cambridge.

Phillips, P. C. B. and Solo, V. (1992) Asymptotics for linear processes. Annals of Statistics 20, 971-1001.

Smith, R. J. and Taylor, A. M. R. (1998) Additional critical values and asymptotic representations for seasonal unit root tests. Journal of Econometrics 85, 269-288.

Xiao, Z. and Phillips, P. C. B. (1998) Higher-order approximations for frequency domain time series regression. Journal of Econometrics 86, 297-336. 


\section{APPENDIX A: SUPPLEMENTARY LEMMAS}

The following lemmas are required for the main proofs in Appendix B.

LEMMA A1. Let $c(z)=\sum_{j=0}^{\infty} c_{j} z^{j}$ with $\sum_{j=0}^{\infty} j^{2} c_{j}^{2}<\infty$. Then:

(a) $c(z)=c(-1)-(1+z) c^{*}(z)$ where $c^{*}(z)=\sum_{j=0}^{\infty} c_{j}^{*} z^{j}, c_{j}^{*}=(-1)^{j} \sum_{k=j+1}^{\infty}(-1)^{k} c_{k}$ and $\sum_{j=0}^{\infty} c_{j}^{* 2}<\infty$.

(b) $c(z)=c\left(e^{i \pi / 2}\right)-\left(1-e^{-i \pi / 2} z\right) \hat{c}(z)$ where $\hat{c}(z)=\sum_{j=0}^{\infty} \hat{c}_{j} z^{j}, \hat{c}_{j}=\sum_{k=j+1}^{\infty} e^{i(k-j) \pi / 2} c_{k}$ and $\sum_{j=0}^{\infty} \hat{c}_{j}^{2}<\infty$.

Proof of Lemma A1. Taking each part in turn:

(a) Note that

$c^{*}(z)=\frac{c(-1)-c(z)}{1+z}=\left[\sum_{k=0}^{\infty}(-1)^{k} c_{k}-\sum_{k=0}^{\infty} c_{k} z^{k}\right] \sum_{k=0}^{\infty}(-1)^{k} z^{k}$.

Picking out the coefficients on the powers of $z$ in the resulting polynomial on the right hand side yields the expressions for the $c_{j}^{*}$. Now

$\sum_{j=0}^{\infty} c_{j}^{* 2}=\sum_{j=0}^{\infty}\left[(-1)^{j} \sum_{k=j+1}^{\infty}(-1)^{k} c_{k}\right]^{2} \leq \sum_{j=0}^{\infty}\left[\sum_{k=j+1}^{\infty}\left|(-1)^{k} c_{k}\right|\right]^{2}=\sum_{j=0}^{\infty}\left[\sum_{k=j+1}^{\infty} k^{a}\left|c_{k}\right| k^{-a}\right]^{2}$

for some suitably chosen constant $a$. The finiteness of the expression on the right hand side of $\sum_{j=0}^{\infty} j^{2} c_{j}^{2}<\infty$ can be shown by following the steps in the proof of Lemma 2.1 on p.987 of Phillips and Solo (1992).

(b) In this case,

$\hat{c}(z)=\frac{c\left(e^{i \pi / 2}\right)-c(z)}{1-e^{-i \pi / 2} z}=\left[\sum_{k=0}^{\infty} e^{i k \pi / 2} c_{k}-\sum_{k=0}^{\infty} c_{k} z^{k}\right] \sum_{k=0}^{\infty} e^{-i k \pi / 2} z^{k}$.

Picking out the coefficients on the powers of $z$ in the resulting polynomial on the right hand side yields the expressions for the $\hat{c}_{j}$. As in part (a),

$\sum_{j=0}^{\infty} \hat{c}_{j}^{2}=\sum_{j=0}^{\infty} e^{-i j \pi}\left[\sum_{k=j+1}^{\infty} e^{i k \pi / 2} c_{k}\right]^{2} \leq \sum_{j=0}^{\infty}\left[\sum_{k=j+1}^{\infty}\left|e^{i k \pi / 2}\right|\left|c_{k}\right|\right]^{2} \leq \sum_{j=0}^{\infty}\left[\sum_{k=j+1}^{\infty} k^{a}\left|c_{k}\right| k^{-a}\right]^{2}$

for some suitably chosen constant $a$. The finiteness of the expression on the right hand side of $\sum_{j=0}^{\infty} j^{2} c_{j}^{2}<\infty$ can once more be shown by following the steps in the proof of Lemma 2.1 on p.987 of Phillips and Solo (1992). 
LEMMA A2. Under Assumption 1:

(a) $T^{-\frac{1}{2}} \sum_{t=1}^{[T r]} \epsilon_{t} \Rightarrow \sigma_{\epsilon} W_{1}(r)$,

(b) $T^{-\frac{1}{2}} \sum_{t=1}^{[T r]}(-1)^{t} \epsilon_{t} \Rightarrow \sigma_{\epsilon} W_{2}(r)$

(c) $T^{-\frac{1}{2}} \sum_{t=1}^{[T r]} e^{ \pm i t \pi / 2} \epsilon_{t} \Rightarrow \frac{\sigma_{\epsilon}}{\sqrt{2}}\left[W_{3}(r) \pm i W_{4}(r)\right]$,

as $T \rightarrow \infty$, where $W_{1}, W_{2}, W_{3}$ and $W_{4}$ are independent Wiener processes on $r \in[0,1]$.

Proof of Lemma A2. Theorem 2.6 of Phillips and Solo (1992) shows that $s_{T}^{-1} S_{[T r]} \Rightarrow$ $W(r)$, a Wiener process, as $T \rightarrow \infty$, if

(i) $s_{T}^{-2} U_{T} \stackrel{p}{\rightarrow} 1$, and

(ii) $\max _{1 \leq t \leq T}\left|\frac{Z_{t}}{s_{T}}\right| \stackrel{p}{\rightarrow} 0$

where $Z_{t}$ denotes the random variable of interest, $S_{T}=\sum_{t=1}^{T} Z_{t}, U_{T}=\sum_{t=1}^{T} Z_{t}^{2}$ and $s_{T}^{2}=$ $E\left(U_{T}\right)=E\left(\sum_{t=1}^{T} Z_{t}^{2}\right)$. Taking each case in turn:

(a) This is demonstrated on p.977 of Phillips and Solo (1992).

(b) Let $Z_{t}=(-1)^{t} \epsilon_{t}$ so that $Z_{t}^{2}=(-1)^{2 t} \epsilon_{t}^{2}=\epsilon_{t}^{2}$. Then $s_{T}^{2}=T \sigma_{\epsilon}^{2}$ under Assumption 1, while $T^{-1} U_{T} \stackrel{p}{\rightarrow} \sigma_{\epsilon}^{2}$ as $T \rightarrow \infty$. Hence (i) holds, while

$\max _{1 \leq t \leq T}\left|\frac{Z_{t}}{s_{T}}\right|=\max _{1 \leq t \leq T}\left|\frac{(-1)^{t} \epsilon_{t}}{\sigma_{\epsilon} \sqrt{T}}\right| \leq \max _{1 \leq t \leq T}\left|\frac{\epsilon_{t}}{\sigma_{\epsilon} \sqrt{T}}\right| \stackrel{p}{\rightarrow} 0$

since $\epsilon_{t} \sim \operatorname{IID}\left(0, \sigma_{\epsilon}^{2}\right)$, thereby verifying (ii).

(c) Now let $Z_{t}=e^{ \pm i t \pi / 2} \epsilon_{t}=\cos (\pi t / 2) \epsilon_{t} \pm i \sin (\pi t / 2) \epsilon_{t} \equiv Z_{R, t} \pm i Z_{I, t}$, and note that $Z_{R, t}$ and $Z_{I, t}$ are independent due to the orthogonality of $\cos (\pi t / 2)$ and $\sin (\pi t / 2)$ for integer $t$. Taking $Z_{R, t}$ first, $s_{R, T}^{2}=E\left(\sum_{t=1}^{T} \cos ^{2}(\pi t / 2) \epsilon_{t}^{2}\right)=\sigma_{\epsilon}^{2} \sum_{t=1}^{T} \cos ^{2}(\pi t / 2)=(T / 2) \sigma_{\epsilon}^{2}$. Since $\cos ^{2}(\pi t / 2)=(1 / 2)[1+\cos (\pi t)]$ it follows that

$\frac{1}{T} \sum_{t=1}^{T} \cos ^{2}(\pi t / 2) \epsilon_{t}^{2}=\frac{1}{2 T} \sum_{t=1}^{T} \epsilon_{t}^{2}+\frac{1}{2 T} \sum_{t=1}^{T} \cos (\pi t) \epsilon_{t}^{2} \stackrel{p}{\rightarrow} \frac{1}{2} \sigma_{\epsilon}^{2}$

as $T \rightarrow \infty$. Hence (i) is satisfied, while

$\max _{1 \leq t \leq T}\left|\frac{\cos (\pi t / 2) \epsilon_{t}}{\left(\sigma_{\epsilon} / \sqrt{2}\right) \sqrt{T}}\right| \leq \max _{1 \leq t \leq T}\left|\frac{\epsilon_{t}}{\left(\sigma_{\epsilon} / \sqrt{2}\right) \sqrt{T}}\right| \stackrel{p}{\rightarrow} 0$

thereby satisfying (ii). Hence $T^{-\frac{1}{2}}\left(\sigma_{\epsilon} / \sqrt{2}\right)^{-1} \sum_{t=1}^{[T r]} \cos (\pi t / 2) \epsilon_{t} \Rightarrow W_{3}(r)$ as required. An almost identical procedure, but using the substitution $\sin ^{2}(\pi t / 2)=(1 / 2)[1-\cos (\pi t)]$, yields 
$T^{-\frac{1}{2}}\left(\sigma_{\epsilon} / \sqrt{2}\right)^{-1} \sum_{t=1}^{[T r]} \sin (\pi t / 2) \epsilon_{t} \Rightarrow W_{4}(r)$.

Finally, the independence of the Wiener processes is a consequence of Theorem 2.2 of Chan and Wei (1988).

\section{APPENDIX B: PROOFS OF LEMMAS AND THEOREMS}

Proof of Lemma 1. The proofs are based on the approach of Phillips and Solo (1992), allied with Lemmas A1 and A2. Taking each part of the Lemma in turn:

(a) This follows directly from Theorem 3.4(b) of Phillips and Solo (1992), the conditions for which are identical to those in Assumption 1 (note that there is a typographical error in the statement of that theorem - the factor $c(1)$ is omitted).

(b) Expanding $c(z)$ around the point $z=-1$ yields $c(z)=c(-1)-(1+z) c^{*}(z)$, where, from Lemma A1, $c^{*}(z)=\sum_{j=0}^{\infty} c_{j}^{*} z^{j}$ with $\sum_{j=0}^{\infty}\left(c_{j}^{*}\right)^{2}<\infty$. Then

$(-1)^{t} u_{t}=(-1)^{t} c(L) \epsilon_{t}=(-1)^{t}\left[c(-1) \epsilon_{t}-\left(\epsilon_{t}^{*}+\epsilon_{t-1}^{*}\right)\right]$

where $\epsilon_{t}^{*}=\sum_{j=0}^{\infty} c_{j}^{*} \epsilon_{t-j}$ has $E\left(\epsilon_{t}^{* 2}\right)<\infty$ by the square summability of the $c_{j}^{*}$. Summing $(-1)^{t} u_{t}$ over $t=1, \ldots, T$ gives

$T^{-\frac{1}{2}} \sum_{t=1}^{T}(-1)^{t} u_{t}=c(-1) T^{-\frac{1}{2}} \sum_{t=1}^{T}(-1)^{t} \epsilon_{t}-T^{-\frac{1}{2}} \sum_{t=1}^{T}(-1)^{t}\left(\epsilon_{t}^{*}+\epsilon_{t-1}^{*}\right)$.

Note that $\sum_{t=1}^{T}(-1)^{t}\left(\epsilon_{t}^{*}+\epsilon_{t-1}^{*}\right)=-\epsilon_{0}^{*}+(-1)^{T} \epsilon_{T}^{*}$. To establish the invariance principle for $(-1)^{t} u_{t}$ it is necessary to establish the convergence in probability to zero of

$\sup _{r}\left|T^{-\frac{1}{2}} \sum_{t=1}^{[T r]}(-1)^{t} u_{t}-c(-1) T^{-\frac{1}{2}} \sum_{t=1}^{[T r]}(-1)^{t} \epsilon_{t}\right| \leq T^{-1 / 2}\left|\epsilon_{0}^{*}\right|+T^{-\frac{1}{2}} \sup _{r}\left|\epsilon_{[T r]}^{*}\right|$.

But the right hand side does converge to zero in probability since $\epsilon_{t}^{*}=O_{p}(1)$ under Assumption 1 and Lemma A1. Hence the stated invariance principle follows from Lemma A2.

(c) Details are given for $e^{i t \pi / 2} u_{t}$ with the result for $e^{-i t \pi / 2} u_{t}$ following as the complex conjugate. Expanding $c(z)$ around the point $z=e^{i \pi / 2}(=i)$ yields $c(z)=c\left(e^{i \pi / 2}\right)-\left(1-e^{i \pi / 2} z\right) \hat{c}(z)$, where, from Lemma A1, $\hat{c}(z)=\sum_{j=0}^{\infty} \hat{c}_{j} z^{j}$ with $\sum_{j=0}^{\infty} \hat{c}_{j}^{2}<\infty$. Then

$e^{i t \pi / 2} u_{t}=e^{i t \pi / 2} c(L) \epsilon_{t}=e^{i t \pi / 2}\left[c\left(e^{i \pi / 2}\right) \epsilon_{t}-\left(\hat{\epsilon}_{t}-e^{-i \pi / 2} \hat{\epsilon}_{t-1}\right)\right]$

where $\hat{\epsilon}_{t}=\sum_{j=0}^{\infty} \hat{c}_{j} \epsilon_{t-j}$ has $E\left(\hat{\epsilon}_{t}^{2}\right)<\infty$ by the square summability of the $\hat{c}_{j}$. Summing $e^{i t \pi / 2} u_{t}$ over $t=1, \ldots, T$ gives

$T^{-\frac{1}{2}} \sum_{t=1}^{T} e^{i t \pi / 2} u_{t}=c\left(e^{i \pi / 2}\right) T^{-\frac{1}{2}} \sum_{t=1}^{T} e^{i t \pi / 2} \epsilon_{t}-T^{-\frac{1}{2}} \sum_{t=1}^{T} e^{i t \pi / 2}\left(\hat{\epsilon}_{t}-e^{-i \pi / 2} \hat{\epsilon}_{t-1}\right)$. 
Note that $\sum_{t=1}^{T} e^{i t \pi / 2}\left(\hat{\epsilon}_{t}-e^{-i \pi / 2} \hat{\epsilon}_{t-1}\right)=-\hat{\epsilon}_{0}+e^{i T \pi / 2} \hat{\epsilon}_{T}$. To establish the invariance principle for $e^{i t \pi / 2} u_{t}$ it is necessary to establish the convergence in probability to zero of $\sup _{r}\left|T^{-\frac{1}{2}} \sum_{t=1}^{[T r]} e^{i t \pi / 2} u_{t}-c\left(e^{i \pi / 2}\right) T^{-\frac{1}{2}} \sum_{t=1}^{[T r]} e^{i t \pi / 2} \epsilon_{t}\right| \leq T^{-1 / 2}\left|\hat{\epsilon}_{0}\right|+T^{-\frac{1}{2}} \sup _{r}\left|\hat{\epsilon}_{[T r]}\right|$.

The convergence in probability of this term to zero follows since $\hat{\epsilon}_{t}=O_{p}(1)$ under Assumption 1 and Lemma A1. Hence the stated invariance principle follows from Lemma A2.

Proof of Lemma 2. (a) From Lemma 1 and the continuous mapping theorem, $T^{-2} \sum_{t=1}^{T} y_{1 t}^{2}=T^{-2} \sum_{t=1}^{T} P_{t}^{2} \Rightarrow \sigma_{\epsilon}^{2} c(1)^{2} \int_{0}^{1} W_{1}^{2}$, while for $n \neq 0$,

$$
\begin{aligned}
T^{-2} \sum_{t=1}^{T-n} y_{1 t} y_{1, t+n} & =T^{-2} \sum_{t=1}^{T-n} y_{1 t}^{2}+T^{-2} \sum_{j=0}^{n-1} \sum_{t=1}^{T-n} y_{1 t} u_{t+n-j} \\
& =T^{-2} \sum_{t=1}^{T-n} P_{t}^{2}+o_{p}(1) \Rightarrow \sigma_{\epsilon}^{2} c(1)^{2} \int_{0}^{1} W_{1}^{2}
\end{aligned}
$$

as required.

(b) Similar arguments can be applied to the autocovariances involving $y_{2 t}$, in which case Lemma 1 and the continuous mapping theorem yield

$T^{-2} \sum_{t=1}^{T} y_{2 t}^{2}=T^{-2} \sum_{t=1}^{T}(-1)^{2 t} M_{t}^{2} \Rightarrow \sigma_{\epsilon}^{2} c(-1)^{2} \int_{0}^{1} W_{2}^{2}$,

while for $n \neq 0$,

$$
\begin{aligned}
T^{-2} \sum_{t=1}^{T-n} y_{2 t} y_{2, t+n} & =T^{-2} \sum_{t=1}^{T-n}(-1)^{t} M_{t}(-1)^{t+n} M_{t+n} \\
& =(-1)^{n} T^{-2} \sum_{t=1}^{T-n} M_{t}^{2}+(-1)^{n} \sum_{j=1}^{n} T^{-2} \sum_{t=1}^{T-n}(-1)^{t+j} M_{t} u_{t+j} \\
& =(-1)^{n} T^{-2} \sum_{t=1}^{T-n} M_{t}^{2}+o_{p}(1) \Rightarrow(-1)^{n} \sigma_{\epsilon}^{2} c(-1)^{2} \int_{0}^{1} W_{2}^{2}
\end{aligned}
$$

as required.

(c) The derivations involving $y_{4 t}$ are rather more involved. From the earlier representation of $y_{4 t}$ in terms of the partial sums $C_{t}$ and $S_{t}$,

$y_{4 t} y_{4, t+n}=C_{t} C_{t+n} \sin [(t+1) \pi / 2] \sin [(t+n+1) \pi / 2]$ 


$$
\begin{aligned}
& +S_{t} S_{t+n} \cos [(t+1) \pi / 2] \cos [(t+n+1) \pi / 2] \\
& -C_{t} S_{t+n} \sin [(t+1) \pi / 2] \cos [(t+n+1) \pi / 2] \\
& -S_{t} C_{t+n} \cos [(t+1) \pi / 2] \sin [(t+n+1) \pi / 2] .
\end{aligned}
$$

The following trigonometric identities are useful:

$$
\begin{aligned}
& \sin [(x \pm y) / 2] \cos [(x \mp y) / 2]=[\sin x \pm \sin y] / 2 \\
& \cos [(x+y) / 2] \cos [(x-y) / 2]=[\cos x+\cos y] / 2 \\
& \sin [(x+y) / 2] \sin [(y-x) / 2]=[\cos x-\cos y] / 2
\end{aligned}
$$

see, for example, equations 1.314.1, 1.314.3, and 1.314.5, respectively, of Gradshteyn and Ryzhik (1994). Setting $x=[t+1+(n / 2)] \pi$ and $y=(n / 2) \pi$ yields

$$
\begin{aligned}
y_{4 t} y_{4, t+n}= & \left.-(1 / 2) C_{t} C_{t+n}\{\cos [(2(t+1)+n) \pi) / 2]-\cos [(n \pi) / 2]\right\} \\
& +(1 / 2) S_{t} S_{t+n}\{\cos [(2(t+1)+n) \pi / 2]+\cos [(n \pi) / 2]\} \\
& -(1 / 2) C_{t} S_{t+n}\{\sin [(2(t+1)+n) \pi / 2]-\sin [(n \pi) / 2]\} \\
& -(1 / 2) S_{t} C_{t+n}\{\sin [(2(t+1)+n) \pi / 2]+\sin [(n \pi) / 2]\},
\end{aligned}
$$

from which it follows that

$$
\begin{aligned}
T^{-2} \sum_{t=1}^{T-n} y_{4 t} y_{4, t+n}= & -\frac{1}{2} T^{-2} \sum_{t=1}^{T-n}\left(C_{t} C_{t+n}-S_{t} S_{t+n}\right) \cos [(2(t+1)+n) \pi / 2] \\
& +\frac{1}{2} T^{-2} \sum_{t=1}^{T-n}\left(C_{t} C_{t+n}+S_{t} S_{t+n}\right) \cos [(n \pi) / 2] \\
& -\frac{1}{2} T^{-2} \sum_{t=1}^{T-n}\left(C_{t} S_{t+n}+S_{t} C_{t+n}\right) \sin [(2(t+1)+n) \pi / 2] \\
& +\frac{1}{2} T^{-2} \sum_{t=1}^{T-n}\left(C_{t} S_{t+n}-S_{t} C_{t+n}\right) \sin [(n \pi) / 2] \\
= & \frac{1}{2} T^{-2} \sum_{t=1}^{T-n}\left(C_{t} C_{t+n}+S_{t} S_{t+n}\right) \cos [(n \pi) / 2] \\
& +\frac{1}{2} T^{-2} \sum_{t=1}^{T-n}\left(C_{t} S_{t+n}-S_{t} C_{t+n}\right) \sin [(n \pi) / 2]+o_{p}(1)
\end{aligned}
$$

the $o_{p}(1)$ terms following by virtue of Lemma 3.3.6 of Chan and Wei (1988). Hence

$$
T^{-2} \sum_{t=1}^{T-n} y_{4 t} y_{4, t+n}= \begin{cases}(-1)^{n / 2} \frac{1}{2} T^{-2} \sum_{t=1}^{T-n}\left(C_{t} C_{t+n}+S_{t} S_{t+n}\right)+o_{p}(1), & n \text { even } \\ (-1)^{(n-1) / 2} \frac{1}{2} T^{-2} \sum_{t=1}^{T-n}\left(C_{t} S_{t+n}-S_{t} C_{t+n}\right)+o_{p}(1), & n \text { odd }\end{cases}
$$


But, since $C_{t+n}=C_{t}+\sum_{j=0}^{n-1} \cos [(t+n-j) \pi / 2] u_{t+n-j}$ and $S_{t+n}=S_{t}+\sum_{j=0}^{n-1} \sin [(t+n-$ $j) \pi / 2] u_{t+n-j}$, it follows that

$$
\begin{aligned}
T^{-2} \sum_{t=1}^{T-n} C_{t} C_{t+n} & =T^{-2} \sum_{t=1}^{T-n} C_{t}^{2}+o_{p}(1), \\
T^{-2} \sum_{t=1}^{T-n} S_{t} S_{t+n} & =T^{-2} \sum_{t=1}^{T-n} S_{t}^{2}+o_{p}(1), \\
T^{-2} \sum_{t=1}^{T-n} C_{t} S_{t+n} & =T^{-2} \sum_{t=1}^{T-n} C_{t} S_{t}+o_{p}(1), \\
T^{-2} \sum_{t=1}^{T-n} S_{t} C_{t+n} & =T^{-2} \sum_{t=1}^{T-n} C_{t} S_{t}+o_{p}(1) .
\end{aligned}
$$

Hence

$T^{-2} \sum_{t=1}^{T-n} y_{4 t} y_{4, t+n}= \begin{cases}(-1)^{n / 2} \frac{1}{2} T^{-2} \sum_{t=1}^{T-n}\left(C_{t}^{2}+S_{t}^{2}\right)+o_{p}(1), & n \text { even, } \\ o_{p}(1), & n \text { odd },\end{cases}$

and so the result in the Lemma follows.

(d), (e) The convergence of the cross autocovariances is a direct consequence of the results on p.379 of Chan and Wei (1988).

Proof of Lemma 3. (a) This is a standard result in the literature; see, for example, Phillips (1987).

(b) Squaring both sides of the equation $M_{t}=M_{t-1}+(-1)^{t} u_{t}$ and summing yields

$$
\begin{aligned}
T^{-1} \sum_{t=1}^{T} M_{t-1}(-1)^{t} u_{t} & =\frac{1}{2} T^{-1} M_{T}^{2}-\frac{1}{2} T^{-1} \sum_{t=1}^{T} u_{t}^{2} \\
& \Rightarrow \frac{1}{2} \sigma_{\epsilon}^{2} c(-1)^{2} W_{2}(1)^{2}-\frac{1}{2} \gamma_{0} \\
& =\frac{1}{2} \sigma_{\epsilon}^{2} c(-1)^{2}\left[W_{2}(1)^{2}-1\right]+\frac{1}{2}\left[\sigma_{\epsilon}^{2} c(-1)^{2}-\gamma_{0}\right] .
\end{aligned}
$$

Noting that $(1 / 2)\left[W_{2}(1)^{2}-1\right]=\int_{0}^{1} W_{2} d W_{2}$ while

$\sigma_{\epsilon}^{2} c(-1)^{2}=2 \pi f_{u u}(\pi)=\sum_{j=-\infty}^{\infty} \gamma_{j} e^{-i j \pi}=\sum_{j=-\infty}^{\infty} \gamma_{j}(-1)^{j}=\gamma_{0}+2 \sum_{j=1}^{\infty}(-1)^{j} \gamma_{j}$

yields the desired result.

(c), (d) These results can be demonstrated jointly by using the results in Phillips (1988). Let $x_{t}=\left(C_{t}, S_{t}\right)^{\prime}$ and $v_{t}=\left[\cos (\pi t / 2) u_{t}, \sin (\pi t / 2) u_{t}\right]^{\prime}$, so that $x_{t}=x_{t-1}+v_{t}$. Further, define $\Gamma_{t, k}=E\left(v_{t} v_{t+k}^{\prime}\right), \Lambda=\lim _{T \rightarrow \infty} T^{-1} \sum_{t=2}^{T} \sum_{k=1}^{t-1} \Gamma_{t, k}, \Sigma=\lim _{T \rightarrow \infty} T^{-1} \sum_{t=1}^{T} \Gamma_{t, 0}$, and $\Omega=\Sigma+\Lambda+\Lambda^{\prime}$. Phillips (1988) shows that $T^{-1} \sum_{t=1}^{T} x_{t-1} v_{t}^{\prime} \Rightarrow \int_{0}^{1} B d B^{\prime}+\Lambda$, where $B$ is 
Brownian motion with variance matrix $\Omega$. Parts (c) and (d) are obtained by selecting the limits of the appropriate elements of

$$
T^{-1} \sum_{t=1}^{T} x_{t-1} v_{t}^{\prime}=\left[\begin{array}{ll}
T^{-1} \sum_{t=1}^{T} C_{t-1} \cos (\pi t / 2) u_{t} & T^{-1} \sum_{t=1}^{T} S_{t-1} \cos (\pi t / 2) u_{t} \\
T^{-1} \sum_{t=1}^{T} C_{t-1} \sin (\pi t / 2) u_{t} & T^{-1} \sum_{t=1}^{T} S_{t-1} \sin (\pi t / 2) u_{t}
\end{array}\right]
$$

and by working out the form of $\Omega$ and $\Lambda$ in this particular case. First note that

$$
\begin{aligned}
& \Gamma_{t, k}=E\left(\begin{array}{cc}
\cos (\pi t / 2) \cos (\pi(t+k) / 2) u_{t} u_{t+k} & \cos (\pi t / 2) \sin (\pi(t+k) / 2) u_{t} u_{t+k} \\
\sin (\pi t / 2) \cos (\pi(t+k) / 2) u_{t} u_{t+k} & \sin (\pi t / 2) \sin (\pi(t+k) / 2) u_{t} u_{t+k}
\end{array}\right) \\
& =\left\{\begin{array}{l}
\left(\begin{array}{cc}
(-1)^{\frac{k}{2}} \gamma_{k} & 0 \\
0 & 0
\end{array}\right), k, t \text { even, } \quad\left(\begin{array}{cc}
0 & (-1)^{\frac{k-1}{2}} \gamma_{k} \\
0 & 0
\end{array}\right), k \text { odd, } t \text { even, } \\
\left(\begin{array}{cc}
0 & 0 \\
0 & (-1)^{\frac{k}{2}} \gamma_{k}
\end{array}\right), k \text { even, } t \text { odd, } \quad\left(\begin{array}{cc}
0 & 0 \\
(-1)^{\frac{k+1}{2}} \gamma_{k} & 0
\end{array}\right), k, t \text { odd. }
\end{array}\right.
\end{aligned}
$$

It then follows that

$$
\begin{aligned}
& \Sigma=\left(\begin{array}{cc}
\frac{1}{2} \gamma_{0} & 0 \\
0 & \frac{1}{2} \gamma_{0}
\end{array}\right), \quad \Lambda=\frac{1}{2}\left(\begin{array}{cc}
\sum_{k=1}^{\infty}(-1)^{k} \gamma_{2 k} & \sum_{k=0}^{\infty}(-1)^{k} \gamma_{2 k+1} \\
-\sum_{k=0}^{\infty}(-1)^{k} \gamma_{2 k+1} & \sum_{k=1}^{\infty}(-1)^{k} \gamma_{2 k}
\end{array}\right), \\
& \Omega=\frac{1}{2}\left(\begin{array}{cc}
\gamma_{0}+2 \sum_{k=1}^{\infty}(-1)^{k} \gamma_{2 k} & 0 \\
0 & \gamma_{0}+2 \sum_{k=1}^{\infty}(-1)^{k} \gamma_{2 k}
\end{array}\right)
\end{aligned}
$$

Finally, noting that

$$
\frac{\sigma_{\epsilon}^{2}}{2}\left|c\left(e^{i \pi / 2}\right)\right|^{2}=\frac{1}{2}\left[2 \pi f_{u u}(\pi / 2)\right]=\frac{1}{2} \sum_{j=-\infty}^{\infty} \gamma_{j} e^{-i j \pi / 2}=\frac{1}{2}\left(\gamma_{0}+2 \sum_{j=1}^{\infty}(-1)^{j} \gamma_{2 j}\right)
$$

yields the stated results.

Proof of Lemma 4. (a) This result is fairly standard in the literature and is given, for example, by Phillips (1991).

(b) Note, first, that

$$
\sum_{t=1}^{T-n} y_{2, t-1} u_{t+n}=(-1)^{n} \sum_{t=1}^{T-n} M_{t-1}\left(M_{t+n}-M_{t+n-1}\right) .
$$

Substituting $M_{t+n}-M_{t+n-1}=M_{t}-M_{t-1}+(-1)^{t+n} u_{t+n}-(-1)^{t} u_{t}$ yields

$$
\begin{aligned}
\sum_{t=1}^{T-n} y_{2, t-1} u_{t+n}= & (-1)^{n} \sum_{t=1}^{T-n} M_{t-1}\left(M_{t}-M_{t-1}\right) \\
& +(-1)^{n} \sum_{t=1}^{T-n} M_{t-1}\left[(-1)^{t+n} u_{t+n}-(-1)^{t} u_{t}\right] .
\end{aligned}
$$


Concentrating on the components of the second term, we obtain

$$
\begin{aligned}
& \sum_{t=1}^{T-n} M_{t-1}(-1)^{t+n} u_{t+n}=\sum_{j=0}^{n}(-1)^{T-j} M_{T-n-1-j} u_{T-j}+\sum_{t=n+1}^{T-n-1} M_{t-n-1}(-1)^{t} u_{t}, \\
& \sum_{t=1}^{T-n} M_{t-1}(-1)^{t} u_{t}=\sum_{t=1}^{T-n} M_{t-n-1}(-1)^{t} u_{t}+\sum_{k=1}^{n}(-1)^{k} \sum_{t=1}^{T-n} u_{t-k} u_{t},
\end{aligned}
$$

so that

$$
\begin{aligned}
& \sum_{t=1}^{T-n} M_{t-1}\left[(-1)^{t+n} u_{t+n}-(-1)^{t} u_{t}\right]=\sum_{j=0}^{n}(-1)^{T-j} M_{T-n-1-j} u_{T-j} \\
& \quad-M_{T-2 n-2}(-1)^{T-n} u_{T-n}-\sum_{t=1}^{n} M_{t-n-1}(-1)^{t} u_{t}-\sum_{k=1}^{n}(-1)^{k} \sum_{t=1}^{T-n} u_{t-k} u_{t} \\
& =\sum_{j=0}^{n}(-1)^{T-j} M_{T-n-1-j} u_{T-j}-M_{T-2 n-2}(-1)^{T-n} u_{T-n}-\sum_{k=1}^{n}(-1)^{k} \sum_{t=1}^{T-n} u_{t-k} u_{t}
\end{aligned}
$$

due to the second term in the second line being null because $M_{t}=0$ for $t \leq 0$. Hence

$$
\begin{aligned}
T^{-1} \sum_{t=1}^{T-n} y_{2, t-1} u_{t+n}= & (-1)^{n} T^{-1} \sum_{t=1}^{T-n} M_{t-1}\left(M_{t}-M_{t-1}\right) \\
& -(-1)^{n} \sum_{k=1}^{n}(-1)^{k} T^{-1} \sum_{t=1}^{T-n} u_{t-k} u_{t}+o_{p}(1)
\end{aligned}
$$

and the result follows.

(c) Some care needs to be taken with respect to the treatment of the term involving $y_{4 t}$, so more detail is provided. From the trigonometric representation of $y_{4 t}$ in the proof of Lemma 2 ,

$$
y_{4, t-1} u_{t+n}=-\left\{C_{t-1} \sin (\pi t / 2) u_{t+n}-S_{t-1} \cos (\pi t / 2) u_{t+n}\right\} .
$$

The following trigonometric identities are useful:

$$
\begin{aligned}
& \sin (x-y)=\sin x \cos y-\sin y \cos x \\
& \cos (x-y)=\cos x \cos y+\sin x \sin y
\end{aligned}
$$

see, for example, equations 1.313.1 and 1.313.5, respectively, of Gradshteyn and Ryzhik (1994). Applying these identities with $x=(t+n) \pi / 2$ and $y=n \pi / 2$ results in

$$
\begin{aligned}
& \sin (\pi t / 2)= \begin{cases}(-1)^{n / 2} \sin [(t+n) \pi / 2], & n \text { even } \\
(-1)^{(n+1) / 2} \cos [(t+n) \pi / 2], & n \text { odd }\end{cases} \\
& \cos (\pi t / 2)= \begin{cases}(-1)^{n / 2} \cos [(t+n) \pi / 2], & n \text { even } \\
(-1)^{(n-1) / 2} \sin [(t+n) \pi / 2], & n \text { odd }\end{cases}
\end{aligned}
$$


Hence, for $n$ even,

$$
\begin{aligned}
y_{4, t-1} u_{t+n} & =-\left\{(-1)^{n / 2} C_{t-1} \sin [(t+n) \pi / 2] u_{t+n}-(-1)^{n / 2} S_{t-1} \cos [(t+n) \pi / 2] u_{t+n}\right\} \\
& =(-1)^{(n+2) / 2}\left[C_{t-1}\left(S_{t+n}-S_{t+n-1}\right)+S_{t-1}\left(C_{t+n}-C_{t+n-1}\right)\right]
\end{aligned}
$$

while for $n$ odd,

$$
\begin{aligned}
y_{4, t-1} u_{t+n}= & -\left\{(-1)^{(n+1) / 2} C_{t-1} \cos [(t+n) \pi / 2] u_{t+n}\right. \\
& \left.-(-1)^{(n-1) / 2} S_{t-1} \sin [(t+n) \pi / 2] u_{t+n}\right\} \\
= & (-1)^{(n-1) / 2}\left[C_{t-1}\left(C_{t+n}-C_{t+n-1}\right)+S_{t-1}\left(S_{t+n}-S_{t+n-1}\right)\right] .
\end{aligned}
$$

The following two substitutions can now be made:

$C_{t+n}-C_{t+n-1}=C_{t}-C_{t-1}+\cos [(t+n) \pi / 2] u_{t+n}-\cos (\pi t / 2) u_{t}$,

$S_{t+n}-S_{t+n-1}=S_{t}-S_{t-1}+\sin [(t+n) \pi / 2] u_{t+n}-\sin (\pi t / 2) u_{t}$.

There are four terms now to consider, all of which can be handled in a similar way. Demonstrating the technique for one of them, we have

$$
\begin{aligned}
\sum_{t=1}^{T-n} C_{t-1}\left[C_{t+n}-C_{t+n-1}\right]= & \sum_{t=1}^{T-n} C_{t-1}\left[C_{t}-C_{t-1}\right] \\
& +\sum_{t=1}^{T-n} C_{t-1}\left[\cos [(t+n) \pi / 2] u_{t+n}-\cos (\pi t / 2) u_{t}\right] .
\end{aligned}
$$

The components of the second term may be written

$$
\begin{aligned}
\sum_{t=1}^{T-n} C_{t-1} \cos [(t+n) \pi / 2] u_{t+n}= & \sum_{j=0}^{n} C_{T-n-1-j} \cos [(T-j) \pi / 2] u_{T-j} \\
& +\sum_{t=n+1}^{T-n-1} C_{t-n-1} \cos (\pi t / 2) u_{t}, \\
\sum_{t=1}^{T-n} C_{t-1} \cos (\pi t / 2) u_{t}= & \sum_{t=1}^{T-n} C_{t-n-1} \cos (\pi t / 2) u_{t} \\
& +\sum_{k=1}^{n} \sum_{t=1}^{T-n} u_{t-k} u_{t} \cos [(t-k) \pi / 2] \cos (\pi t / 2) .
\end{aligned}
$$

Defining $c_{t+n}=\cos [(t+n) \pi / 2]$ and $s_{t+n}=\sin [(t+n) \pi / 2]$ in order to save some space,

$$
T^{-1} \sum_{t=1}^{T-n} C_{t-1}\left[c_{t+n} u_{t+n}-c_{t} u_{t}\right]=-\sum_{k=1}^{n} T^{-1} \sum_{t=1}^{T-n} u_{t-k} u_{t} c_{t-k} c_{t}+o_{p}(1) .
$$


Similarly,

$$
\begin{aligned}
T^{-1} \sum_{t=1}^{T-n} S_{t-1}\left[s_{t+n} u_{t+n}-s_{t} u_{t}\right] & =-\sum_{k=1}^{n} T^{-1} \sum_{t=1}^{T-n} u_{t-k} u_{t} s_{t-k} s_{t}+o_{p}(1), \\
T^{-1} \sum_{t=1}^{T-n} C_{t-1}\left[s_{t+n} u_{t+n}-s_{t} u_{t}\right] & =-\sum_{k=1}^{n} T^{-1} \sum_{t=1}^{T-n} u_{t-k} u_{t} c_{t-k} s_{t}+o_{p}(1), \\
T^{-1} \sum_{t=1}^{T-n} S_{t-1}\left[c_{t+n} u_{t+n}-c_{t} u_{t}\right] & =-\sum_{k=1}^{n} T^{-1} \sum_{t=1}^{T-n} u_{t-k} u_{t} s_{t-k} c_{t}+o_{p}(1) .
\end{aligned}
$$

Utilising these decompositions we obtain, for $n$ even,

$$
\begin{aligned}
T^{-1} \sum_{t=1}^{T-n} y_{4, t-1} u_{t+n}= & (-1)^{(n+2) / 2} T^{-1}\left[\sum_{t=1}^{T-n} C_{t-1}\left(S_{t}-S_{t-1}\right)-\sum_{t=1}^{T-n} S_{t-1}\left(C_{t}-C_{t-1}\right)\right] \\
& -(-1)^{(n+2) / 2} \sum_{k=1}^{n} T^{-1} \sum_{t=1}^{T-n} u_{t-k} u_{t}\left[c_{t-k} s_{t}-s_{t-k} c_{t}\right]+o_{p}(1),
\end{aligned}
$$

while for $n$ odd,

$$
\begin{aligned}
T^{-1} \sum_{t=1}^{T-n} y_{4, t-1} u_{t+n}= & (-1)^{(n-1) / 2} T^{-1}\left[\sum_{t=1}^{T-n} C_{t-1}\left(C_{t}-C_{t-1}\right)+\sum_{t=1}^{T-n} S_{t-1}\left(S_{t}-S_{t-1}\right)\right] \\
& -(-1)^{(n-1) / 2} \sum_{k=1}^{n} T^{-1} \sum_{t=1}^{T-n} u_{t-k} u_{t}\left[c_{t-k} c_{t}+s_{t-k} s_{t}\right]+o_{p}(1) .
\end{aligned}
$$

Application of the results in Lemma 3 yields the required expressions.

Proof of Theorem 1. It is convenient to define

$$
H_{M}=\frac{1}{2 M} \sum_{j=-M+1}^{M} \hat{f}_{x x}\left(\omega_{j}\right) f_{u u}\left(\omega_{j}\right)^{-1} \text { and } h_{M}=\frac{1}{2 M} \sum_{j=-M+1}^{M} \hat{f}_{x u}\left(\omega_{j}\right) f_{u u}\left(\omega_{j}\right)^{-1} \text {. }
$$

From the definition of $\hat{f}_{x x}(\lambda)$ in (7) and making use of the Fourier series representation of $f_{u u}(\lambda)^{-1}$, given by

$f_{u u}(\lambda)^{-1}=\frac{1}{2 \pi} \sum_{g=-\infty}^{\infty} d_{g} e^{i g \lambda}, \quad-\pi<\lambda \leq \pi$,

it is possible to write

$$
T^{-1} H_{M}=\left(\frac{1}{2 \pi}\right)^{2} \sum_{g=-\infty}^{\infty} d_{g} \sum_{n=-M}^{M} k\left(\frac{n}{M}\right) T^{-1} C_{x x}(n) \frac{1}{2 M} \sum_{j=-M+1}^{M} e^{i(g-n) \pi j / M} .
$$


Now $\sum_{j=-M+1}^{M} e^{i(g-n) \pi j / M}=2 M$ if $(g-n)= \pm 2 l M$ for integer $l$, and is equal to zero otherwise. Hence the non-zero terms determining $H_{M}$ are those for which $n=g+2 l M$ $(l=0, \pm 1, \ldots)$, and we may write

$T^{-1} H_{M}=\left(\frac{1}{2 \pi}\right)^{2} \sum_{g=-\infty}^{\infty} d_{g} \sum_{l=-\infty}^{\infty} k\left(\frac{g+2 l M}{M}\right) T^{-1} C_{x x}(g+2 l M) ;$

see Hannan (1963), Phillips (1991) and Choi and Phillips (1993) for alternative representations. Note that this expression only includes those $C_{x x}(n)$ for $-M \leq n \leq M$ because $k(x)=0$ for $|x|>1$ i.e. $k(n / M)=0$ if $n=g+2 l M>M$. As $T \rightarrow \infty$ and hence $M \rightarrow \infty$, $k((g+2 l M) / M) \rightarrow k(2 l)$ which is zero for all $l \neq 0$, and so it is only the $l=0$ term that is influential. Hence we are led to consider

$T^{-1} H_{M}=\left(\frac{1}{2 \pi}\right)^{2} \sum_{g=-\infty}^{\infty} d_{g} T^{-1} C_{x x}(g)+o_{p}(1)$

since $k(0)=1$ under Assumption 2. The limiting behaviour of the elements of $T^{-1} C_{x x}(g)$ is provided in Lemma 2, which shows that all off-diagonal elements, with the exception of those corresponding to $y_{3}$ and $y_{4}$, converge to zero. It is therefore necessary to demonstrate that the diagonal elements of $T^{-1} H_{M}$ converge to the stated limits, while the cross-product terms involving $y_{3}$ and $y_{4}$ converge to zero.

$H_{11}$ : From Lemma 2 we obtain

$T^{-1} H_{M, 11} \Rightarrow \sigma_{\epsilon}^{2} c(1)^{2} \int_{0}^{1} W_{1}^{2}\left(\frac{1}{2 \pi}\right)^{2} \sum_{g=-\infty}^{\infty} d_{g}=\int_{0}^{1} W_{1}^{2}$,

since $(1 / 2 \pi)^{2} \sum_{g} d_{g}=(1 / 2 \pi) f_{u u}(0)^{-1}=1 / \sigma^{2}$ where $\sigma^{2}=\sigma_{\epsilon}^{2} c(1)^{2}$ denotes the long run variance of $u_{t}$.

$\underline{H_{22}}$ : Here, the limit depends on $g$, so that

$T^{-1} H_{M, 22} \Rightarrow \sigma_{\epsilon}^{2} c(-1)^{2} \int_{0}^{1} W_{2}^{2}\left(\frac{1}{2 \pi}\right)^{2} \sum_{g=-\infty}^{\infty} d_{g}(-1)^{g}=\int_{0}^{1} W_{2}^{2}$,

since $(1 / 2 \pi) \sigma_{\epsilon}^{2} c(-1)^{2}=f_{u u}(\pi)$ and $(1 / 2 \pi) \sum_{g} d_{g}(-1)^{g}=(1 / 2 \pi) \sum_{g} d_{g} e^{i g \pi}=f_{u u}(\pi)^{-1}$.

$H_{33}, H_{44}$ : That $T^{-1} H_{M, 33}$ and $T^{-1} H_{M, 44}$ converge to the same limit arises because

$T^{-2} \sum_{t} y_{3 t} y_{3, t+n}=T^{-2} \sum_{t} y_{4, t-1} y_{4, t-1+n}=T^{-2} \sum_{t} y_{4 t} y_{4, t+n}+o_{p}(1)$.

Concentrating on $T^{-1} H_{M, 44}$ we obtain

$T^{-1} H_{M, 44} \Rightarrow \frac{\sigma_{\epsilon}^{2}}{4}\left|c\left(e^{i \pi / 2}\right)\right|^{2} \int_{0}^{1}\left(W_{3}^{2}+W_{4}^{2}\right)\left(\frac{1}{2 \pi}\right)^{2} \sum_{g \text { even }} d_{g}(-1)^{g / 2}$.

Noting that $f_{u u}(\pi / 2)=(1 / 2 \pi) \sigma_{\epsilon}^{2}\left|c\left(e^{i \pi / 2}\right)\right|^{2}$ and that

$f_{u u}(\pi / 2)^{-1}=\frac{1}{2 \pi} \sum_{g} d_{g} e^{i g \pi / 2}=\frac{1}{2 \pi} \sum_{g \text { even }} d_{g}(-1)^{g / 2}$ 
yields the stated result.

Cross-products, $y_{3}$ and $y_{4}$ : Lemma 2 implies that, since $y_{3 t}=y_{4, t-1}$,

$T^{-2} \sum_{t=1}^{T-n} y_{3 t} y_{4, t+n} \Rightarrow\left\{\begin{array}{l}0, \quad n \text { even, } \\ (-1)^{(n+1) / 2} \frac{\sigma_{\epsilon}^{2}}{4}\left|c\left(e^{i \pi / 2}\right)\right|^{2} \int_{0}^{1}\left[W_{3}^{2}+W_{4}^{2}\right], \quad n \text { odd. }\end{array}\right.$

Hence

$T^{-1} H_{M, 34} \Rightarrow \frac{\sigma_{\epsilon}^{2}}{4}\left|c\left(e^{i \pi / 2}\right)\right|^{2} \int_{0}^{1}\left(W_{3}^{2}+W_{4}^{2}\right)\left(\frac{1}{2 \pi}\right)^{2} \sum_{g \text { odd }} d_{g}(-1)^{(g+1) / 2}=0$

the last summation being null since $d_{g}=d_{-g}$ and hence $d_{g}(-1)^{(g+1) / 2}=-d_{-g}(-1)^{(-g+1) / 2}$. Cross-products, $y_{4}$ and $y_{3}$ : This follows in a similar fashion to $H_{34}$, but note that

$T^{-2} \sum_{t=1}^{T-n} y_{4 t} y_{3, t+n} \Rightarrow\left\{\begin{array}{l}0, \quad n \text { even, } \\ (-1)^{(n-1) / 2} \frac{\sigma_{\epsilon}^{2}}{4}\left|c\left(e^{i \pi / 2}\right)\right|^{2} \int_{0}^{1}\left[W_{3}^{2}+W_{4}^{2}\right], \quad n \text { odd. }\end{array}\right.$

since $T^{-2} \sum_{t=1}^{T-n} y_{4 t} y_{3, t+n}=T^{-2} \sum_{t=1}^{T-n} y_{4 t} y_{4, t-1+n}$. Hence

$T^{-1} H_{M, 34} \Rightarrow \frac{\sigma_{\epsilon}^{2}}{4}\left|c\left(e^{i \pi / 2}\right)\right|^{2} \int_{0}^{1}\left(W_{3}^{2}+W_{4}^{2}\right)\left(\frac{1}{2 \pi}\right)^{2} \sum_{g \text { odd }} d_{g}(-1)^{(g-1) / 2}=0$

by the same argument as before.

This concludes the proof of the limiting form of $T^{-1} H_{M}$.

Turning to $h_{M}$, similar arguments lead to the expression

$h_{M}=\left(\frac{1}{2 \pi}\right)^{2} \sum_{g=-\infty}^{\infty} d_{g} C_{x u}(g)+o_{p}(1)$

and the convergence of $h_{m}$ to the limits stated in the Theorem will be demonstrated using the results of Lemma 4. Taking each element in turn:

$\underline{h_{1}}$ : From Lemma 4,

$$
\begin{aligned}
h_{M, 1} & \Rightarrow \sigma_{\epsilon}^{2} c(1)^{2} \int_{0}^{1} W_{1} d W_{1}\left(\frac{1}{2 \pi}\right)^{2} \sum_{g=-\infty}^{\infty} d_{g}+\left(\frac{1}{2 \pi}\right)^{2} \sum_{g=-\infty}^{\infty} d_{g} \delta_{1}(g+1) \\
& =\int_{0}^{1} W_{1} d W_{1}+\left(\frac{1}{2 \pi}\right)^{2} \sum_{g=-\infty}^{\infty} d_{g} \delta_{1}(g+1) .
\end{aligned}
$$

Note that $\delta_{1}(g+1)=\sum_{j=0}^{\infty} E u_{0} u_{j+g+1}$, and so we may write, as in Phillips (1991),

$$
\sum_{g=-\infty}^{\infty} d_{g} \delta_{1}(g+1)=\sum_{g=-\infty}^{\infty}\left[\sum_{j=0}^{\infty} E u_{0} u_{j+g+1}\right] d_{g}=\sum_{j=0}^{\infty} E u_{0} v_{j}
$$


where $v_{j}=\sum_{g=-\infty}^{\infty} u_{j+g+1} d_{g}$. Now $E u_{0} v_{j}=\int_{-\pi}^{\pi} e^{i j \lambda} f_{u v}(\lambda) d \lambda$, where $f_{u v}(\lambda)$ is the cross spectral density between $u$ and $v$ and is given by

$$
\begin{aligned}
f_{u v}(\lambda)=\frac{1}{2 \pi} \sum_{k=-\infty}^{\infty} e^{-i k \lambda} E u_{0} v_{k} & =\frac{1}{2 \pi} \sum_{k=-\infty}^{\infty} e^{-i k \lambda} E u_{0} \sum_{g=-\infty}^{\infty} u_{k+g+1} d_{g} \\
& =\frac{1}{2 \pi} \sum_{m=-\infty}^{\infty} E u_{0} u_{m} e^{-i m \lambda} \sum_{g=-\infty}^{\infty} d_{g} e^{i(g+1) \lambda} \\
& =f_{u u}(\lambda) e^{i \lambda} 2 \pi f_{u u}(\lambda)^{-1}=2 \pi e^{i \lambda},
\end{aligned}
$$

the second line following by substituting $m=k+g+1$. This implies that

$E u_{0} v_{j}=2 \pi \int_{-\pi}^{\pi} e^{i(j+1) \lambda} d \lambda= \begin{cases}(2 \pi)^{2}, & j=-1 \\ 0 & \text { otherwise }\end{cases}$

and hence

$\sum_{g=-\infty}^{\infty} d_{g} \delta_{1}(g+1)=\sum_{j=0}^{\infty} E u_{0} v_{j}=0$,

from which it follows that $h_{1}=\int_{0}^{1} W_{1} d W_{1}$ as required.

$\underline{h_{2}}$ : Again from Lemma 4,

$$
\begin{aligned}
h_{M, 2} & \Rightarrow \sigma_{\epsilon}^{2} c(-1)^{2} \int_{0}^{1} W_{2} d W_{2}\left(\frac{1}{2 \pi}\right)^{2} \sum_{g=-\infty}^{\infty} d_{g}(-1)^{g}+\left(\frac{1}{2 \pi}\right)^{2} \sum_{g=-\infty}^{\infty} d_{g}(-1)^{g} \delta_{2}(g+1) \\
& =\int_{0}^{1} W_{2} d W_{2}+\left(\frac{1}{2 \pi}\right)^{2} \sum_{g=-\infty}^{\infty} d_{g}(-1)^{g} \delta_{2}(g+1) .
\end{aligned}
$$

Recall that $\delta_{2}(g+1)=\sum_{j=0}^{\infty}(-1)^{j+g+1} E u_{0} u_{j+g+1}$, so that

$$
\begin{aligned}
\sum_{g=-\infty}^{\infty} d_{g}(-1)^{g} \delta_{2}(g+1) & =\sum_{g=-\infty}^{\infty}(-1)^{g}\left[\sum_{j=0}^{\infty}(-1)^{j+g+1} E u_{0} u_{j+g+1}\right] d_{g} \\
& =\sum_{j=0}^{\infty}(-1)^{j+1} E u_{0} \sum_{g=-\infty}^{\infty}(-1)^{2 g} u_{j+g+1} d_{g} \\
& =\sum_{j=0}^{\infty}(-1)^{j+1} E u_{0} v_{j}=0
\end{aligned}
$$

in view of the earlier results concerning $E u_{0} v_{j}$. Hence $h_{2}=\int_{0}^{1} W_{2} d W_{2}$ as required. $\underline{h_{3}, h_{4}}$ : These two terms are similar, in view of Lemma $4(\mathrm{c})$. Considering $h_{4}$,

$$
\begin{aligned}
h_{M, 4} \Rightarrow & \frac{\sigma_{\epsilon}^{2}}{2}\left|c\left(e^{i \pi / 2}\right)\right|^{2} \int_{0}^{1}\left(W_{3} d W_{4}-W_{4} d W_{3}\right)\left(\frac{1}{2 \pi}\right)^{2} \sum_{g \text { even }} d_{g}(-1)^{(g+2) / 2} \\
& +\frac{\sigma_{\epsilon}^{2}}{2}\left|c\left(e^{i \pi / 2}\right)\right|^{2} \int_{0}^{1}\left(W_{3} d W_{3}+W_{4} d W_{4}\right)\left(\frac{1}{2 \pi}\right)^{2} \sum_{g \text { odd }} d_{g}(-1)^{(g-1) / 2}
\end{aligned}
$$




$$
\begin{aligned}
& +\left(\frac{1}{2 \pi}\right)^{2} \sum_{g \text { even }} d_{g}(-1)^{(g+2) / 2} \delta_{4}(g)+\left(\frac{1}{2 \pi}\right)^{2} \sum_{g \text { odd }} d_{g}(-1)^{(g-1) / 2} \delta_{3}(g) \\
= & -\frac{1}{2} \int_{0}^{1}\left(W_{3} d W_{4}-W_{4} d W_{3}\right)+\left(\frac{1}{2 \pi}\right)^{2} \sum_{g=-\infty}^{\infty} d_{g}(-1)^{\left[\frac{g+1}{2}\right]+1} \delta_{5}(g),
\end{aligned}
$$

where $\delta_{5}(g)=\sum_{j=0}^{\infty}(-1)^{j+\left[\frac{g+1}{2}\right]} E u_{0} u_{j+g+1}$. This last term is

$$
\begin{aligned}
\sum_{g=-\infty}^{\infty}(-1)^{\left[\frac{g+1}{2}\right]+1}\left[\sum_{j=0}^{\infty}(-1)^{j+\left[\frac{g+1}{2}\right]} E u_{0} u_{j+g+1}\right] d_{g} & =\sum_{j=0}^{\infty}(-1)^{j+1} E u_{0} \sum_{g=-\infty}^{\infty} u_{j+g+1} d_{g} \\
& =\sum_{j=0}^{\infty}(-1)^{j+1} E u_{0} v_{j}=0
\end{aligned}
$$

as before, since the factor $(-1)^{2\left[\frac{g+1}{2}\right]}=1$ for all $g$ in the second expression in the first line. Hence $h_{4}=(1 / 2) \int_{0}^{1}\left(W_{4} d W_{3}-W_{3} d W_{4}\right)$ as required. Similar arguments yield the expression for $h_{3}$ given in the Theorem.

Proof of Theorem 2. It is convenient to begin by deriving the limiting distribution of $T(\hat{\beta}-\beta)$. Note that

$T(\hat{\beta}-\beta)=\left[\frac{1}{2 M} \sum_{j=-M+1}^{M} \hat{f}_{x x}\left(\omega_{j}\right) \hat{f}_{\hat{u} \hat{u}}\left(\omega_{j}\right)^{-1}\right]^{-1}\left[\frac{1}{2 M} \sum_{j=-M+1}^{M} \hat{f}_{x \hat{u}}\left(\omega_{j}\right) \hat{f}_{\hat{u} \hat{u}}\left(\omega_{j}\right)^{-1}\right]$.

Since $\hat{\beta}_{O L S}$ is a consistent estimator of $\beta$ it can be shown that under Assumptions 1 and 2

$\max _{\lambda}\left|\hat{f}_{\hat{u} \hat{u}}(\lambda)-f_{u u}(\lambda)\right| \stackrel{p}{\rightarrow} 0$

as $T \rightarrow \infty$, and so to examine the asymptotics it is sufficient to focus on

$T(\hat{\beta}-\beta)=\left[T^{-1} H_{M}\right]^{-1} h_{M}$,

where $H_{M}$ and $h_{M}$ are defined in the proof of Theorem 1. The diagonality of the limit of $T^{-1} H_{M}$ and the fact that $\beta=0$ under the null yields immediately that $T \hat{\beta}_{j} \Rightarrow h_{j} / H_{j j}$ $(j=1, \ldots, 4)$, giving the expressions in the Theorem.

Turning to the t-ratios, the limiting distributions are obtained by multiplying the limiting distributions of the $T \hat{\beta}_{j}$ by the square roots of the appropriate diagonal elements of the limit of $T^{-1} H_{M}$ (since $T^{2} V_{T}=T H_{M}^{-1}$ can be regarded as the variance matrix of $T \hat{\beta}$ ). The diagonal elements of interest follow from Theorem 1 and the above proof.

The limiting distributions of the Wald statistics follow in a similar way. Since it is possible to write

$J_{i}=T \hat{\beta}_{i}^{\prime} R_{i}^{\prime}\left[R_{i}\left(T^{-1} H_{M}\right)^{-1} R_{i}^{\prime}\right]^{-1} R_{i} T \hat{\beta}$, 
and the limiting distributions of $T \hat{\beta}$ and $T^{-1} H_{M}$ have already been established, it is straightforward to show that $J_{i}$ converges to the limits stated in the Lemma.

Proof of Corollary to Theorem 2. Consider the case where $\beta_{1}=0$ but $\beta_{j} \neq 0$ $(j=2,3,4)$. From (2) and (3) it follows that $\left(1-L^{4}\right) w_{t}=\sum_{j=2}^{4} \beta_{j} \alpha_{j}(L) w_{t-1}+u_{t}$ or $w_{t}=\sum_{j=2}^{4} \beta_{j} \frac{\alpha_{j}(L)}{1-L^{4}} w_{t-1}+\frac{u_{t}}{1-L^{4}}$.

From the definitions of the polynomials $\alpha_{j}(z)$ following (3),

$$
\frac{\alpha_{2}(z)}{1-z^{4}}=\frac{-1}{1+z} ; \quad \frac{\alpha_{3}(z)}{1-z^{4}}=\frac{-z}{1+z^{2}} ; \quad \frac{\alpha_{4}(z)}{1-z^{4}}=\frac{-1}{1+z^{2}} .
$$

Since $y_{1 t}=\alpha_{1}(L) w_{t}$ the above decompositions yield

$y_{1 t}=\alpha_{1}(L)\left[-\frac{\beta_{2}}{1+L}-\frac{\beta_{3} L}{1+L^{2}}-\frac{\beta_{4}}{1+L^{2}}\right] w_{t-1}+\frac{\alpha_{1}(L)}{1-L^{4}} u_{t}$.

But $\alpha_{1}(z)=(1+z)\left(1+z^{2}\right)$ and $\left(1-z^{4}\right)=(1-z) \alpha_{1}(z)$, so that

$y_{1 t}=-\beta_{2}\left(1+L^{2}\right) w_{t-1}-\beta_{3}(1+L) w_{t-2}-\beta_{4}(1+L) w_{t-1}+P_{t}$,

where $P_{t}=(1-L)^{-1} u_{t}=O_{p}\left(t^{\frac{1}{2}}\right)$. Hence $y_{1 t}=P_{t}+O_{p}(1)$ and

$\frac{1}{T^{2}} \sum_{t=1}^{T} y_{1 t}^{2}=\frac{1}{T^{2}} \sum_{t=1}^{T} P_{t}^{2}+o_{p}(1) \Rightarrow \sigma_{\epsilon}^{2} c(1)^{2} \int_{0}^{1} W_{1}^{2}$

as before. By similar arguments, $y_{2 t}=-(-1)^{t} M_{t}+O_{p}(1)$ when $\beta_{j} \neq 0(j \neq 2)$ and $y_{4 t}=-\left[C_{t} \sin (t+1) \pi / 2-S_{t} \cos (t+1) \pi / 2\right]+O_{p}(1)$ when $\beta_{j} \neq 0(j \neq 4)$, so that the limits in Lemma 2, for example, apply. The analysis also extends to Lemmas 3 and 4. 
Table 1. Size of Frequency Domain Test Statistics Based on Tukey Kernel

\begin{tabular}{|c|c|c|c|c|c|c|c|c|c|c|c|}
\hline$T$ & $\psi$ & $T \hat{\beta}_{1}$ & $T \hat{\beta}_{2}$ & $T \hat{\beta}_{3}$ & $T \hat{\beta}_{4}$ & $t_{\beta_{1}}$ & $t_{\beta_{2}}$ & $t_{\beta_{3}}$ & $t_{\beta_{4}}$ & $J_{1234}$ & $J_{34}$ \\
\hline & & \multicolumn{10}{|c|}{$\delta=1 / 5$} \\
\hline \multirow[t]{5}{*}{48} & 0.5 & 0.177 & 0.005 & 0.113 & 0.005 & 0.140 & 0.003 & 0.082 & 0.004 & 0.139 & 0.088 \\
\hline & 0.2 & 0.091 & 0.028 & 0.065 & 0.026 & 0.068 & 0.020 & 0.046 & 0.020 & 0.043 & 0.042 \\
\hline & 0.0 & 0.052 & 0.052 & 0.052 & 0.058 & 0.040 & 0.040 & 0.038 & 0.043 & 0.035 & 0.036 \\
\hline & -0.2 & 0.028 & 0.095 & 0.065 & 0.108 & 0.022 & 0.071 & 0.047 & 0.069 & 0.046 & 0.042 \\
\hline & -0.5 & 0.004 & 0.179 & 0.112 & 0.230 & 0.003 & 0.140 & 0.078 & 0.124 & 0.137 & 0.085 \\
\hline \multirow[t]{5}{*}{100} & 0.5 & 0.149 & 0.004 & 0.108 & 0.006 & 0.128 & 0.003 & 0.088 & 0.007 & 0.130 & 0.090 \\
\hline & 0.2 & 0.086 & 0.025 & 0.058 & 0.025 & 0.071 & 0.022 & 0.046 & 0.026 & 0.050 & 0.049 \\
\hline & 0.0 & 0.051 & 0.050 & 0.048 & 0.052 & 0.044 & 0.043 & 0.040 & 0.046 & 0.040 & 0.043 \\
\hline & -0.2 & 0.024 & 0.087 & 0.062 & 0.091 & 0.021 & 0.077 & 0.051 & 0.068 & 0.052 & 0.050 \\
\hline & -0.5 & 0.004 & 0.153 & 0.106 & 0.202 & 0.003 & 0.134 & 0.085 & 0.127 & 0.132 & 0.086 \\
\hline \multirow[t]{6}{*}{200} & 0.5 & 0.128 & 0.004 & 0.104 & 0.009 & 0.116 & 0.003 & 0.091 & 0.088 & 0.114 & 0.083 \\
\hline & 0.2 & 0.078 & 0.026 & 0.063 & 0.030 & 0.072 & 0.025 & 0.057 & 0.028 & 0.053 & 0.054 \\
\hline & 0.0 & 0.047 & 0.052 & 0.050 & 0.052 & 0.044 & 0.050 & 0.047 & 0.048 & 0.043 & 0.043 \\
\hline & -0.2 & 0.026 & 0.081 & 0.061 & 0.090 & 0.025 & 0.074 & 0.057 & 0.069 & 0.052 & 0.050 \\
\hline & -0.5 & 0.004 & 0.127 & 0.101 & 0.180 & 0.004 & 0.115 & 0.089 & 0.114 & 0.109 & 0.081 \\
\hline & & \multicolumn{10}{|c|}{$\delta=1 / 3$} \\
\hline \multirow[t]{5}{*}{48} & 0.5 & 0.172 & 0.005 & 0.109 & 0.007 & 0.137 & 0.004 & 0.078 & 0.007 & 0.120 & 0.075 \\
\hline & 0.2 & 0.090 & 0.030 & 0.066 & 0.030 & 0.068 & 0.021 & 0.048 & 0.023 & 0.040 & 0.041 \\
\hline & 0.0 & 0.054 & 0.052 & 0.053 & 0.055 & 0.042 & 0.040 & 0.040 & 0.044 & 0.034 & 0.037 \\
\hline & -0.2 & 0.031 & 0.089 & 0.066 & 0.096 & 0.023 & 0.068 & 0.046 & 0.066 & 0.044 & 0.041 \\
\hline & -0.5 & 0.006 & 0.170 & 0.107 & 0.190 & 0.003 & 0.135 & 0.077 & 0.105 & 0.119 & 0.073 \\
\hline \multirow[t]{5}{*}{100} & 0.5 & 0.140 & 0.009 & 0.095 & 0.015 & 0.120 & 0.007 & 0.075 & 0.015 & 0.104 & 0.067 \\
\hline & 0.2 & 0.080 & 0.032 & 0.058 & 0.034 & 0.069 & 0.027 & 0.047 & 0.034 & 0.048 & 0.050 \\
\hline & 0.0 & 0.055 & 0.053 & 0.053 & 0.052 & 0.047 & 0.045 & 0.044 & 0.049 & 0.043 & 0.046 \\
\hline & -0.2 & 0.032 & 0.083 & 0.065 & 0.077 & 0.027 & 0.073 & 0.052 & 0.060 & 0.052 & 0.050 \\
\hline & -0.5 & 0.008 & 0.144 & 0.093 & 0.142 & 0.006 & 0.123 & 0.073 & 0.094 & 0.104 & 0.065 \\
\hline \multirow[t]{5}{*}{200} & 0.5 & 0.108 & 0.012 & 0.078 & 0.025 & 0.096 & 0.010 & 0.068 & 0.024 & 0.076 & 0.059 \\
\hline & 0.2 & 0.066 & 0.036 & 0.060 & 0.041 & 0.062 & 0.033 & 0.054 & 0.037 & 0.051 & 0.051 \\
\hline & 0.0 & 0.045 & 0.053 & 0.047 & 0.052 & 0.041 & 0.051 & 0.045 & 0.050 & 0.045 & 0.045 \\
\hline & -0.2 & 0.035 & 0.070 & 0.058 & 0.067 & 0.032 & 0.065 & 0.053 & 0.054 & 0.048 & 0.047 \\
\hline & -0.5 & 0.014 & 0.111 & 0.076 & 0.104 & 0.011 & 0.099 & 0.067 & 0.070 & 0.074 & 0.055 \\
\hline
\end{tabular}


Table 2. Size of Frequency Domain Test Statistics Based on Parzen Kernel

\begin{tabular}{|c|c|c|c|c|c|c|c|c|c|c|c|}
\hline$T$ & $\psi$ & $T \hat{\beta}_{1}$ & $T \hat{\beta}_{2}$ & $T \hat{\beta}_{3}$ & $T \hat{\beta}_{4}$ & $t_{\beta_{1}}$ & $t_{\beta_{2}}$ & $t_{\beta_{3}}$ & $t_{\beta_{4}}$ & $J_{1234}$ & $J_{34}$ \\
\hline & & \multicolumn{10}{|c|}{$\delta=1 / 5$} \\
\hline \multirow[t]{5}{*}{48} & 0.5 & 0.216 & 0.003 & 0.121 & 0.002 & 0.173 & 0.003 & 0.087 & 0.002 & 0.178 & 0.111 \\
\hline & 0.2 & 0.104 & 0.025 & 0.064 & 0.021 & 0.077 & 0.018 & 0.046 & 0.018 & 0.047 & 0.043 \\
\hline & 0.0 & 0.054 & 0.053 & 0.051 & 0.055 & 0.042 & 0.041 & 0.037 & 0.044 & 0.033 & 0.036 \\
\hline & -0.2 & 0.026 & 0.107 & 0.064 & 0.118 & 0.020 & 0.079 & 0.047 & 0.081 & 0.049 & 0.046 \\
\hline & -0.5 & 0.003 & 0.215 & 0.121 & 0.282 & 0.002 & 0.171 & 0.086 & 0.168 & 0.181 & 0.109 \\
\hline \multirow[t]{5}{*}{100} & 0.5 & 0.201 & 0.002 & 0.134 & 0.002 & 0.175 & 0.001 & 0.111 & 0.003 & 0.196 & 0.134 \\
\hline & 0.2 & 0.101 & 0.021 & 0.062 & 0.021 & 0.087 & 0.017 & 0.053 & 0.021 & 0.058 & 0.054 \\
\hline & 0.0 & 0.052 & 0.049 & 0.052 & 0.055 & 0.044 & 0.042 & 0.044 & 0.049 & 0.039 & 0.043 \\
\hline & -0.2 & 0.021 & 0.102 & 0.068 & 0.121 & 0.018 & 0.086 & 0.058 & 0.086 & 0.060 & 0.055 \\
\hline & -0.5 & 0.002 & 0.203 & 0.130 & 0.294 & 0.002 & 0.177 & 0.109 & 0.197 & 0.198 & 0.127 \\
\hline \multirow[t]{6}{*}{200} & 0.5 & 0.194 & 0.002 & 0.120 & 0.002 & 0.179 & 0.002 & 0.108 & 0.003 & 0.195 & 0.131 \\
\hline & 0.2 & 0.096 & 0.022 & 0.062 & 0.019 & 0.091 & 0.021 & 0.057 & 0.021 & 0.065 & 0.060 \\
\hline & 0.0 & 0.048 & 0.054 & 0.048 & 0.049 & 0.045 & 0.052 & 0.045 & 0.047 & 0.044 & 0.044 \\
\hline & -0.2 & 0.022 & 0.101 & 0.061 & 0.107 & 0.020 & 0.093 & 0.056 & 0.085 & 0.064 & 0.056 \\
\hline & -0.5 & 0.002 & 0.185 & 0.119 & 0.273 & 0.003 & 0.171 & 0.108 & 0.189 & 0.196 & 0.130 \\
\hline & & \multicolumn{10}{|c|}{$\delta=1 / 3$} \\
\hline \multirow[t]{5}{*}{48} & 0.5 & 0.185 & 0.004 & 0.115 & 0.004 & 0.147 & 0.003 & 0.082 & 0.004 & 0.146 & 0.094 \\
\hline & 0.2 & 0.093 & 0.027 & 0.065 & 0.023 & 0.069 & 0.019 & 0.045 & 0.019 & 0.043 & 0.042 \\
\hline & 0.0 & 0.053 & 0.050 & 0.052 & 0.053 & 0.040 & 0.039 & 0.037 & 0.043 & 0.035 & 0.036 \\
\hline & -0.2 & 0.028 & 0.094 & 0.065 & 0.102 & 0.021 & 0.071 & 0.046 & 0.071 & 0.046 & 0.043 \\
\hline & -0.5 & 0.003 & 0.183 & 0.114 & 0.232 & 0.002 & 0.145 & 0.079 & 0.129 & 0.144 & 0.089 \\
\hline \multirow[t]{5}{*}{100} & 0.5 & 0.143 & 0.005 & 0.107 & 0.010 & 0.124 & 0.004 & 0.086 & 0.009 & 0.121 & 0.082 \\
\hline & 0.2 & 0.082 & 0.027 & 0.059 & 0.029 & 0.069 & 0.023 & 0.048 & 0.028 & 0.050 & 0.049 \\
\hline & 0.0 & 0.052 & 0.050 & 0.050 & 0.055 & 0.044 & 0.043 & 0.042 & 0.049 & 0.042 & 0.045 \\
\hline & -0.2 & 0.026 & 0.084 & 0.064 & 0.090 & 0.022 & 0.074 & 0.053 & 0.067 & 0.052 & 0.050 \\
\hline & -0.5 & 0.005 & 0.148 & 0.104 & 0.187 & 0.004 & 0.128 & 0.084 & 0.118 & 0.121 & 0.078 \\
\hline \multirow[t]{5}{*}{200} & 0.5 & 0.113 & 0.007 & 0.088 & 0.015 & 0.102 & 0.006 & 0.076 & 0.015 & 0.087 & 0.061 \\
\hline & 0.2 & 0.072 & 0.032 & 0.060 & 0.033 & 0.066 & 0.029 & 0.054 & 0.032 & 0.050 & 0.050 \\
\hline & 0.0 & 0.046 & 0.053 & 0.048 & 0.048 & 0.044 & 0.050 & 0.045 & 0.048 & 0.044 & 0.041 \\
\hline & -0.2 & 0.032 & 0.073 & 0.058 & 0.072 & 0.030 & 0.068 & 0.053 & 0.060 & 0.048 & 0.045 \\
\hline & -0.5 & 0.008 & 0.115 & 0.085 & 0.127 & 0.007 & 0.101 & 0.073 & 0.083 & 0.083 & 0.060 \\
\hline
\end{tabular}


Table 3. Size of Time Domain (HEGY) Test Statistics

\begin{tabular}{rrrrcccc}
\hline \multirow{2}{*}{$T$} & $\psi$ & $t_{\beta_{1}}$ & $t_{\beta_{2}}$ & $t_{\beta_{3}}$ & $t_{\beta_{4}}$ & $F_{1234}$ & $F_{34}$ \\
\hline \multirow{2}{*}{48} & 0.5 & 0.037 & 0.040 & 0.043 & 0.026 & 0.046 & 0.039 \\
& 0.2 & 0.040 & 0.042 & 0.043 & 0.029 & 0.042 & 0.037 \\
& 0.0 & 0.040 & 0.040 & 0.040 & 0.038 & 0.047 & 0.042 \\
& -0.2 & 0.044 & 0.039 & 0.043 & 0.040 & 0.047 & 0.040 \\
& -0.5 & 0.042 & 0.039 & 0.042 & 0.047 & 0.047 & 0.039 \\
100 & 0.5 & 0.044 & 0.054 & 0.050 & 0.037 & 0.055 & 0.049 \\
& 0.2 & 0.043 & 0.046 & 0.049 & 0.041 & 0.046 & 0.049 \\
& 0.0 & 0.044 & 0.045 & 0.046 & 0.045 & 0.048 & 0.047 \\
& -0.2 & 0.043 & 0.051 & 0.053 & 0.046 & 0.050 & 0.046 \\
& -0.5 & 0.046 & 0.048 & 0.047 & 0.061 & 0.048 & 0.047 \\
200 & 0.5 & 0.046 & 0.047 & 0.047 & 0.037 & 0.047 & 0.049 \\
& 0.2 & 0.047 & 0.046 & 0.051 & 0.041 & 0.048 & 0.049 \\
& 0.0 & 0.043 & 0.048 & 0.047 & 0.049 & 0.046 & 0.044 \\
& -0.2 & 0.046 & 0.049 & 0.049 & 0.052 & 0.045 & 0.045 \\
& -0.5 & 0.054 & 0.045 & 0.045 & 0.064 & 0.046 & 0.043 \\
\hline
\end{tabular}


Table 4. Power of Frequency Domain Test Statistics Based on Tukey Kernel

\begin{tabular}{|c|c|c|c|c|c|c|c|c|c|c|c|}
\hline$T$ & $\psi$ & $T \hat{\beta}_{1}$ & $T \hat{\beta}_{2}$ & $T \hat{\beta}_{3}$ & $T \hat{\beta}_{4}$ & $t_{\beta_{1}}$ & $t_{\beta_{2}}$ & $t_{\beta_{3}}$ & $t_{\beta_{4}}$ & $J_{1234}$ & $J_{34}$ \\
\hline & & \multicolumn{10}{|c|}{$\delta=1 / 5$} \\
\hline \multirow[t]{5}{*}{48} & 0.5 & 0.351 & 0.007 & 0.378 & 0.008 & 0.299 & 0.009 & 0.307 & 0.002 & 0.340 & 0.276 \\
\hline & 0.2 & 0.185 & 0.054 & 0.241 & 0.042 & 0.157 & 0.049 & 0.193 & 0.013 & 0.136 & 0.116 \\
\hline & 0.0 & 0.103 & 0.105 & 0.223 & 0.107 & 0.094 & 0.095 & 0.190 & 0.044 & 0.107 & 0.095 \\
\hline & -0.2 & 0.050 & 0.192 & 0.249 & 0.231 & 0.044 & 0.162 & 0.202 & 0.090 & 0.137 & 0.121 \\
\hline & -0.5 & 0.007 & 0.355 & 0.379 & 0.501 & 0.009 & 0.300 & 0.304 & 0.234 & 0.338 & 0.281 \\
\hline \multirow[t]{5}{*}{100} & 0.5 & 0.550 & 0.013 & 0.755 & 0.006 & 0.515 & 0.020 & 0.712 & 0.001 & 0.713 & 0.616 \\
\hline & 0.2 & 0.339 & 0.111 & 0.566 & 0.054 & 0.324 & 0.120 & 0.538 & 0.012 & 0.478 & 0.339 \\
\hline & 0.0 & 0.218 & 0.221 & 0.538 & 0.157 & 0.221 & 0.229 & 0.522 & 0.046 & 0.449 & 0.297 \\
\hline & -0.2 & 0.110 & 0.347 & 0.568 & 0.340 & 0.114 & 0.334 & 0.546 & 0.124 & 0.488 & 0.353 \\
\hline & -0.5 & 0.013 & 0.562 & 0.749 & 0.682 & 0.018 & 0.531 & 0.704 & 0.367 & 0.707 & 0.608 \\
\hline \multirow[t]{6}{*}{200} & 0.5 & 0.881 & 0.052 & 0.996 & 0.004 & 0.874 & 0.076 & 0.994 & 0.001 & 0.993 & 0.965 \\
\hline & 0.2 & 0.715 & 0.335 & 0.975 & 0.068 & 0.724 & 0.374 & 0.969 & 0.007 & 0.973 & 0.845 \\
\hline & 0.0 & 0.552 & 0.542 & 0.969 & 0.220 & 0.585 & 0.569 & 0.962 & 0.048 & 0.969 & 0.806 \\
\hline & -0.2 & 0.337 & 0.700 & 0.976 & 0.491 & 0.376 & 0.709 & 0.969 & 0.163 & 0.973 & 0.843 \\
\hline & -0.5 & 0.054 & 0.885 & 0.995 & 0.875 & 0.078 & 0.875 & 0.993 & 0.551 & 0.994 & 0.966 \\
\hline & & \multicolumn{10}{|c|}{$\delta=1 / 3$} \\
\hline \multirow[t]{5}{*}{48} & 0.5 & 0.343 & 0.008 & 0.360 & 0.011 & 0.290 & 0.010 & 0.295 & 0.003 & 0.298 & 0.242 \\
\hline & 0.2 & 0.183 & 0.054 & 0.243 & 0.045 & 0.156 & 0.050 & 0.198 & 0.014 & 0.123 & 0.110 \\
\hline & 0.0 & 0.107 & 0.103 & 0.228 & 0.104 & 0.097 & 0.094 & 0.194 & 0.043 & 0.101 & 0.095 \\
\hline & -0.2 & 0.055 & 0.186 & 0.252 & 0.211 & 0.047 & 0.155 & 0.208 & 0.085 & 0.127 & 0.116 \\
\hline & -0.5 & 0.009 & 0.335 & 0.364 & 0.443 & 0.011 & 0.286 & 0.294 & 0.200 & 0.294 & 0.243 \\
\hline \multirow[t]{5}{*}{100} & 0.5 & 0.503 & 0.023 & 0.691 & 0.016 & 0.467 & 0.029 & 0.645 & 0.002 & 0.612 & 0.492 \\
\hline & 0.2 & 0.320 & 0.133 & 0.569 & 0.072 & 0.310 & 0.138 & 0.540 & 0.018 & 0.455 & 0.315 \\
\hline & 0.0 & 0.222 & 0.227 & 0.555 & 0.160 & 0.228 & 0.233 & 0.534 & 0.049 & 0.445 & 0.295 \\
\hline & -0.2 & 0.129 & 0.328 & 0.578 & 0.299 & 0.132 & 0.314 & 0.548 & 0.107 & 0.462 & 0.330 \\
\hline & -0.5 & 0.022 & 0.512 & 0.689 & 0.561 & 0.027 & 0.477 & 0.641 & 0.269 & 0.610 & 0.492 \\
\hline \multirow[t]{5}{*}{200} & 0.5 & 0.803 & 0.111 & 0.985 & 0.019 & 0.789 & 0.135 & 0.976 & 0.002 & 0.978 & 0.889 \\
\hline & 0.2 & 0.653 & 0.403 & 0.966 & 0.109 & 0.661 & 0.439 & 0.962 & 0.016 & 0.967 & 0.809 \\
\hline & 0.0 & 0.539 & 0.542 & 0.963 & 0.227 & 0.569 & 0.571 & 0.958 & 0.052 & 0.967 & 0.794 \\
\hline & -0.2 & 0.389 & 0.643 & 0.965 & 0.401 & 0.426 & 0.657 & 0.960 & 0.122 & 0.966 & 0.806 \\
\hline & -0.5 & 0.106 & 0.811 & 0.981 & 0.710 & 0.129 & 0.800 & 0.977 & 0.345 & 0.978 & 0.887 \\
\hline
\end{tabular}


Table 5. Power of Frequency Domain Test Statistics Based on Parzen Kernel

\begin{tabular}{|c|c|c|c|c|c|c|c|c|c|c|c|}
\hline$T$ & $\psi$ & $T \hat{\beta}_{1}$ & $T \hat{\beta}_{2}$ & $T \hat{\beta}_{3}$ & $T \hat{\beta}_{4}$ & $t_{\beta_{1}}$ & $t_{\beta_{2}}$ & $t_{\beta_{3}}$ & $t_{\beta_{4}}$ & $J_{1234}$ & $J_{34}$ \\
\hline & & \multicolumn{10}{|c|}{$\delta=1 / 5$} \\
\hline \multirow[t]{5}{*}{48} & 0.5 & 0.408 & 0.006 & 0.391 & 0.005 & 0.348 & 0.008 & 0.319 & 0.001 & 0.411 & 0.323 \\
\hline & 0.2 & 0.209 & 0.051 & 0.240 & 0.034 & 0.174 & 0.048 & 0.192 & 0.011 & 0.147 & 0.124 \\
\hline & 0.0 & 0.107 & 0.110 & 0.218 & 0.102 & 0.097 & 0.097 & 0.187 & 0.043 & 0.107 & 0.096 \\
\hline & -0.2 & 0.049 & 0.212 & 0.247 & 0.247 & 0.044 & 0.176 & 0.202 & 0.104 & 0.148 & 0.131 \\
\hline & -0.5 & 0.007 & 0.410 & 0.394 & 0.561 & 0.009 & 0.347 & 0.321 & 0.297 & 0.408 & 0.330 \\
\hline \multirow[t]{5}{*}{100} & 0.5 & 0.644 & 0.009 & 0.804 & 0.002 & 0.607 & 0.014 & 0.774 & 0.001 & 0.816 & 0.709 \\
\hline & 0.2 & 0.377 & 0.097 & 0.593 & 0.044 & 0.360 & 0.107 & 0.576 & 0.009 & 0.521 & 0.369 \\
\hline & 0.0 & 0.223 & 0.221 & 0.560 & 0.161 & 0.224 & 0.225 & 0.553 & 0.046 & 0.461 & 0.303 \\
\hline & -0.2 & 0.098 & 0.381 & 0.599 & 0.394 & 0.104 & 0.364 & 0.586 & 0.158 & 0.527 & 0.382 \\
\hline & -0.5 & 0.009 & 0.657 & 0.799 & 0.795 & 0.016 & 0.623 & 0.771 & 0.497 & 0.817 & 0.707 \\
\hline \multirow[t]{6}{*}{200} & 0.5 & 0.952 & 0.034 & 0.997 & 0.001 & 0.947 & 0.062 & 0.996 & 0.000 & 0.998 & 0.986 \\
\hline & 0.2 & 0.773 & 0.306 & 0.974 & 0.045 & 0.778 & 0.352 & 0.969 & 0.004 & 0.980 & 0.866 \\
\hline & 0.0 & 0.565 & 0.556 & 0.965 & 0.215 & 0.598 & 0.586 & 0.960 & 0.045 & 0.972 & 0.812 \\
\hline & -0.2 & 0.307 & 0.766 & 0.975 & 0.561 & 0.354 & 0.771 & 0.969 & 0.209 & 0.980 & 0.865 \\
\hline & -0.5 & 0.039 & 0.952 & 0.997 & 0.948 & 0.065 & 0.947 & 0.995 & 0.725 & 0.999 & 0.986 \\
\hline & & \multicolumn{10}{|c|}{$\delta=1 / 3$} \\
\hline \multirow[t]{5}{*}{48} & 0.5 & 0.362 & 0.007 & 0.381 & 0.006 & 0.307 & 0.009 & 0.306 & 0.002 & 0.350 & 0.285 \\
\hline & 0.2 & 0.189 & 0.051 & 0.242 & 0.038 & 0.159 & 0.047 & 0.191 & 0.012 & 0.137 & 0.117 \\
\hline & 0.0 & 0.104 & 0.103 & 0.222 & 0.100 & 0.093 & 0.093 & 0.187 & 0.043 & 0.105 & 0.096 \\
\hline & -0.2 & 0.050 & 0.191 & 0.250 & 0.223 & 0.043 & 0.161 & 0.200 & 0.092 & 0.138 & 0.124 \\
\hline & -0.5 & 0.007 & 0.359 & 0.383 & 0.500 & 0.009 & 0.305 & 0.304 & 0.241 & 0.351 & 0.292 \\
\hline \multirow[t]{5}{*}{100} & 0.5 & 0.530 & 0.015 & 0.742 & 0.009 & 0.497 & 0.021 & 0.703 & 0.001 & 0.682 & 0.581 \\
\hline & 0.2 & 0.329 & 0.115 & 0.573 & 0.062 & 0.316 & 0.124 & 0.550 & 0.015 & 0.476 & 0.335 \\
\hline & 0.0 & 0.216 & 0.221 & 0.548 & 0.163 & 0.220 & 0.226 & 0.536 & 0.048 & 0.456 & 0.299 \\
\hline & -0.2 & 0.113 & 0.335 & 0.578 & 0.333 & 0.119 & 0.321 & 0.561 & 0.121 & 0.484 & 0.349 \\
\hline & -0.5 & 0.015 & 0.539 & 0.734 & 0.653 & 0.019 & 0.507 & 0.695 & 0.337 & 0.681 & 0.576 \\
\hline \multirow[t]{5}{*}{200} & 0.5 & 0.842 & 0.076 & 0.992 & 0.011 & 0.827 & 0.100 & 0.986 & 0.001 & 0.985 & 0.929 \\
\hline & 0.2 & 0.688 & 0.370 & 0.970 & 0.087 & 0.693 & 0.407 & 0.964 & 0.010 & 0.969 & 0.819 \\
\hline & 0.0 & 0.557 & 0.541 & 0.964 & 0.218 & 0.583 & 0.570 & 0.958 & 0.050 & 0.968 & 0.795 \\
\hline & -0.2 & 0.375 & 0.665 & 0.970 & 0.435 & 0.410 & 0.677 & 0.963 & 0.135 & 0.968 & 0.818 \\
\hline & -0.5 & 0.083 & 0.840 & 0.990 & 0.789 & 0.103 & 0.830 & 0.986 & 0.422 & 0.985 & 0.929 \\
\hline
\end{tabular}


Table 6. Power of Time Domain (HEGY) Test Statistics

\begin{tabular}{rrrrcccc}
\hline \multirow{2}{*}{$T$} & $\psi$ & $t_{\beta_{1}}$ & $t_{\beta_{2}}$ & $t_{\beta_{3}}$ & $t_{\beta_{4}}$ & $F_{1234}$ & $F_{34}$ \\
\hline \multirow{2}{*}{48} & 0.5 & 0.095 & 0.092 & 0.166 & 0.006 & 0.411 & 0.323 \\
& 0.2 & 0.094 & 0.089 & 0.182 & 0.017 & 0.139 & 0.094 \\
& 0.0 & 0.095 & 0.092 & 0.196 & 0.035 & 0.144 & 0.102 \\
& -0.2 & 0.091 & 0.096 & 0.192 & 0.060 & 0.142 & 0.104 \\
& -0.5 & 0.094 & 0.094 & 0.165 & 0.097 & 0.145 & 0.099 \\
100 & 0.5 & 0.213 & 0.220 & 0.443 & 0.004 & 0.500 & 0.318 \\
& 0.2 & 0.208 & 0.223 & 0.522 & 0.016 & 0.483 & 0.301 \\
& 0.0 & 0.213 & 0.225 & 0.557 & 0.043 & 0.501 & 0.317 \\
& -0.2 & 0.195 & 0.228 & 0.521 & 0.092 & 0.496 & 0.314 \\
& -0.5 & 0.198 & 0.231 & 0.434 & 0.191 & 0.496 & 0.313 \\
200 & 0.5 & 0.567 & 0.520 & 0.830 & 0.001 & 0.971 & 0.803 \\
& 0.2 & 0.571 & 0.551 & 0.936 & 0.010 & 0.972 & 0.800 \\
& 0.0 & 0.565 & 0.557 & 0.960 & 0.047 & 0.974 & 0.807 \\
& -0.2 & 0.550 & 0.548 & 0.939 & 0.130 & 0.972 & 0.800 \\
& -0.5 & 0.527 & 0.571 & 0.828 & 0.334 & 0.976 & 0.803 \\
\hline
\end{tabular}




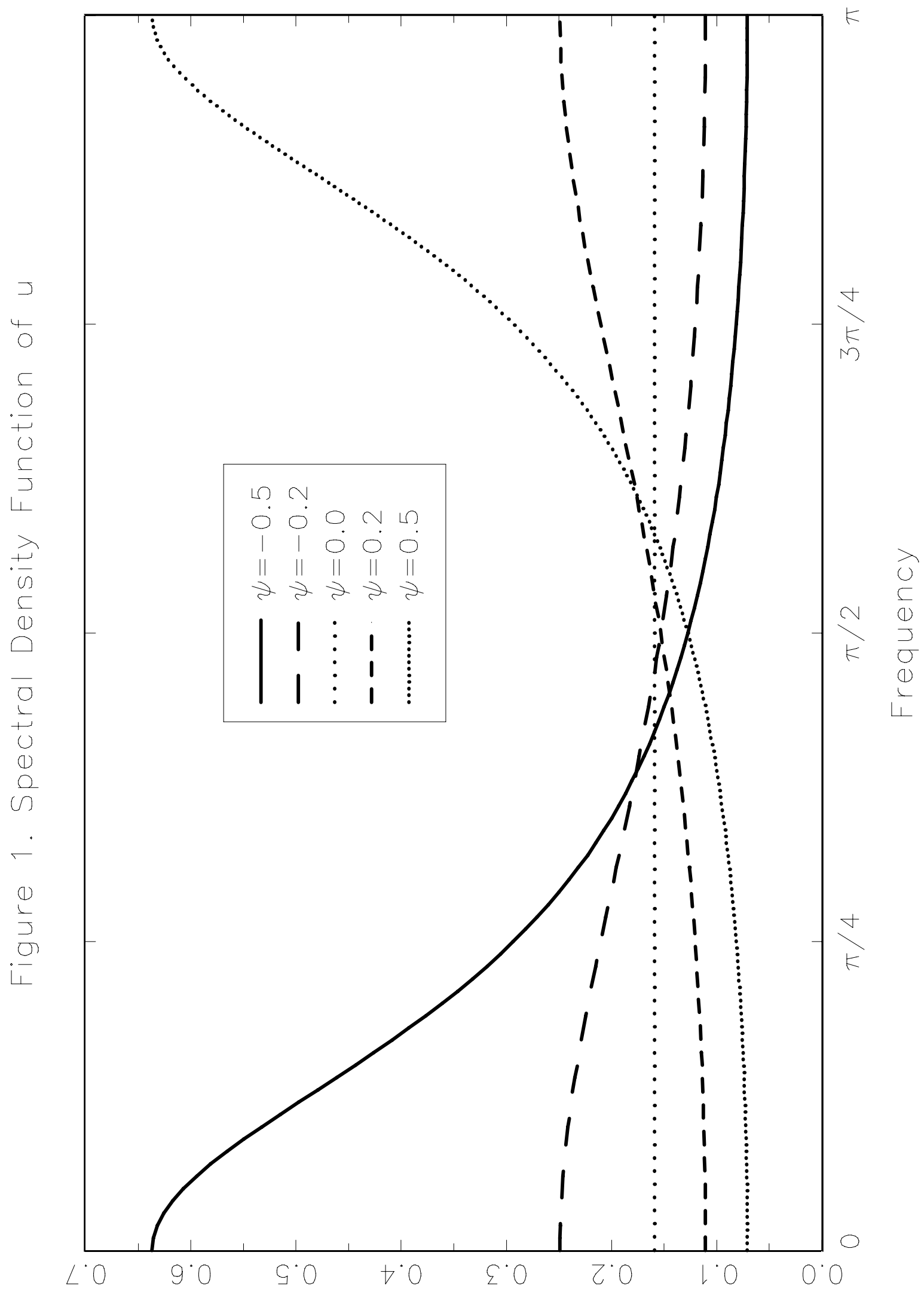




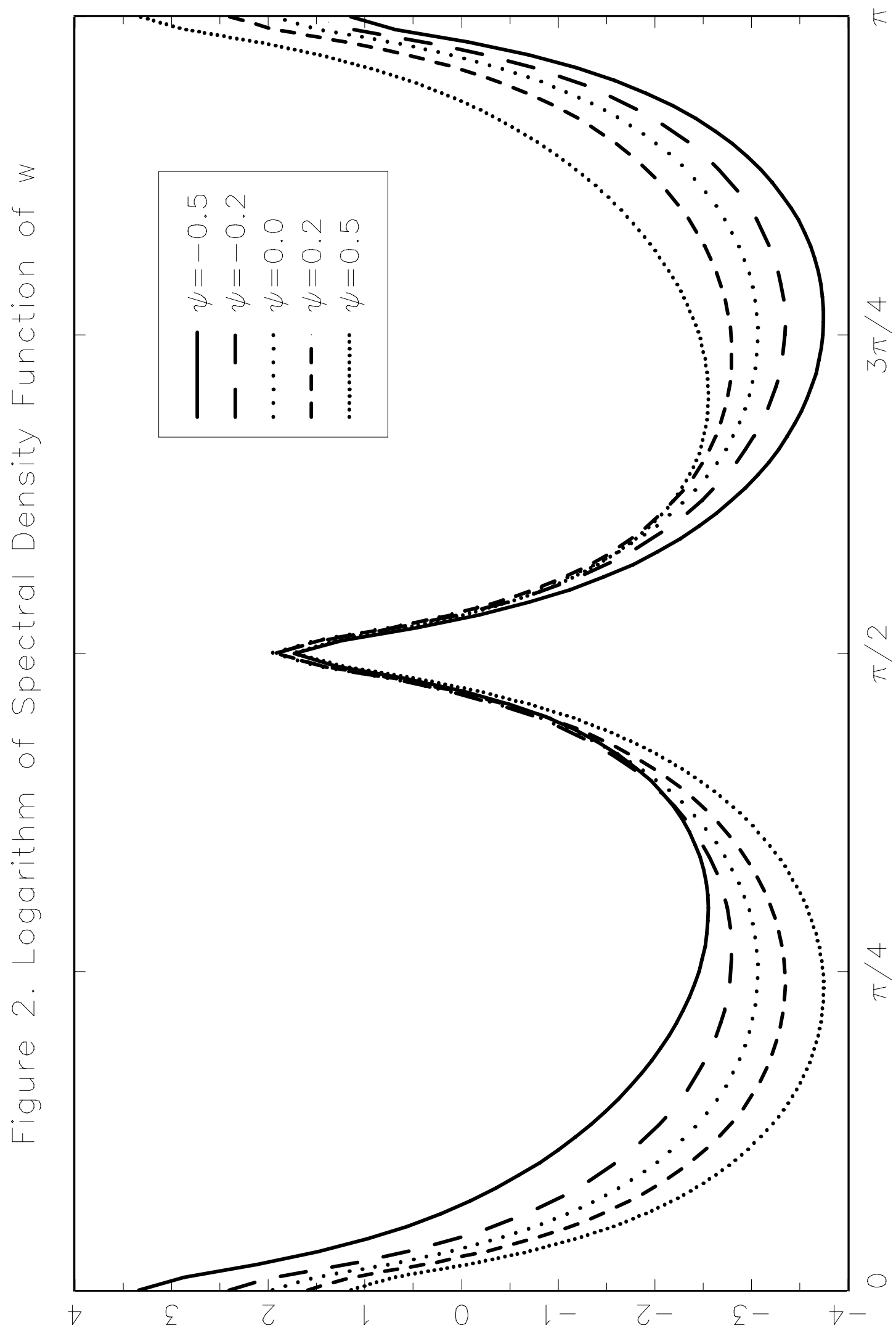

